\title{
Thermodynamic properties of an integrable quantum spin ladder with boundary impurities
}

\author{
M.T. Batchelor ${ }^{\mathrm{a}, \mathrm{b}}, \mathrm{X} .-\mathrm{W}$. Guan $^{\mathrm{a}, \mathrm{b}}$, A. Foerster ${ }^{\mathrm{c}}$, A.P. Tonel ${ }^{\mathrm{c}}$, \\ H.-Q. Zhou ${ }^{\mathrm{d}}$ \\ ${ }^{a}$ Department of Theoretical Physics, Research School of Physical Sciences and \\ Engineering, Australian National University, Canberra ACT 0200, Australia \\ ${ }^{\mathrm{b}}$ Centre for Mathematics and its applications, School of Mathematical Sciences, \\ Australian National University, Canberra ACT 0200, Australia \\ ${ }^{\mathrm{c}}$ Instituto de Fisica da UFRGS, Av. Bento Goncalves, 9500, \\ Porto Alegre, 91501-970, Brazil \\ ${ }^{\mathrm{d}}$ Centre for Mathematical Physics, School of Physical Sciences, \\ The University of Queensland, 4072, Australia
}

\begin{abstract}
An integrable quantum spin ladder based on the SU(4) symmetry algebra with boundary defects is studied in the framework of boundary integrability. Five nontrivial solutions of the reflection equations lead to different boundary impurities. In each case the energy spectrum is determined using the quantum inverse scattering method. The thermodynamic properties are investigated by means of the thermodynamic Bethe ansatz. In particular, the susceptibility and the magnetization of the model in the vicinity of the critical points are derived along with differing magnetic properites for antiferromagnetic and ferromagnetic impurity couplings at the edges. The results are applicable to the strong coupling ladder compounds, such as $\mathrm{Cu}_{2}\left(\mathrm{C}_{5} \mathrm{H}_{12} \mathrm{~N}_{2}\right)_{2} \mathrm{Cl}_{4}$.
\end{abstract}

Key words: Yang-Baxter equation; reflection equations; thermodynamic Bethe ansatz; impurity effects, quantum spin ladders

PACS: 75.10.Jm; 75.30.Kz; 75.40.Cx

\section{Introduction}

Research on quantum spin ladders continues to attract considerable attention from both theoretical and experimental points of view due to their relevance 
to a large number of low-dimensional materials, such as particular cuprates and organic compounds $[1,2,3,4,5,6]$, among others. Initially, most of the theoretical results concerning ladder systems were obtained from the standard Heisenberg ladder. Subsequently, other models with generalized interactions have been proposed. In this context, Nersesyan and Tsvelik [7] introduced a spin ladder model incorporating a biquadratic spin exchange interaction term, which, when sufficiently strong, exhibits new dimerized phases [8]. Various ladder models have been developed by an extension of the symmetry algebra $[9,10,11,12,13]$. A special case of the Nersesyan-Tsvelik model [7] was proposed later by Wang [12]. This model, based on the $S U(4)$ symmetry algebra, is exactly solvable by Bethe ansatz methods and exhibits a spin gap in the spectrum of elementary triplet excitations, a necessary condition for superconductivity to occur under hole doping. In addition, it was recently observed [14] that this model can be used to describe some physical properties of different types of two-leg ladder compounds, such as $\mathrm{Cu}_{2}\left(\mathrm{C}_{5} \mathrm{H}_{12} \mathrm{~N}_{2}\right)_{2} \mathrm{Cl}_{4}$ [3], $\left(\mathrm{C}_{5} \mathrm{H}_{12} \mathrm{~N}\right)_{2} \mathrm{CuBr}_{4}$ [4], (5IAP) $)_{2} \mathrm{CuBr}_{4} \cdot 2 \mathrm{H}_{2} \mathrm{O}$ [5] and $\mathrm{KCuCl}_{3}, \mathrm{TlCuCl}_{3}$ [6]. In the absence of a magnetic field the model exhibits three quantum phases, while in the presence of a strong magnetic field there is a gapped phase in the regime $H<H_{c 1}$, a fully polarized gapped phase for $H>H_{c 2}$ and a Luttinger liquid magnetic phase in the regime $H_{c 1}<H<H_{c 2}$. This observation suggests that the physical properties of the ladder compounds can be accessed via the well-established knowledge of integrable systems.

On the other hand, the effect of boundary impurities and defects also plays an important role in quasi-one dimensional systems. An integrable $S U(4)$ spin ladder model with a boundary defect has been proposed and investigated recently through Bethe ansatz methods [15]. This model, however, is just a particular case of a more general family of exactly solvable ladder models based on the $S U(4)$ symmetry algebra that can be constructed from more general types of bounday conditions. Basically, by this strategy, a set of equations to deal with the boundaries, called reflection equations (RE) are introduced $[16,17]$. The solutions of these equations $[18,19,20]$, referred to as $K$-matrices, in turn introduce boundary interactions into the Hamiltonian of the system, in such a manner that integrability is preserved. The boundary interaction terms in spin ladder models may be realized by impurity doping at the ends of the ladder. Impurity doping in a spin ladder system with a spin gap has been performed [21]. Substantial change in macroscopic properties such as enhancement in spin correlations and magnetic susceptibilities are observed in the low impurity concentration region. The boundary impurity doping may change the critical behaviour at the boundaries of the ladder systems.

The purpose of this paper is to present a complete family of integrable spin ladder systems based on the $S U(4)$ symmetry algebra with boundary impurities in a systematic way. An analytic analysis of the thermodynamic properties of these models is then performed by means of the thermodynamic Bethe ansatz 
(TBA) method and, in particular, the effect of these impurities on the free energy, the susceptibility and the magnetization is discussed. So far, the results obtained provide a clear interpretation of the impurity effects in the low temperature regime of an integrable open spin ladder system.

The paper is organized as follows. In section 2, we present the $\mathrm{SU}(4)$ solution of the YBE and solve the corresponding RE. Furthermore, we give the explicit expressions for the Hamiltonian with different types of boundary defects. Section 3 is devoted to the derivation of the Bethe-ansatz solution by means of the Quantum Inverse Scattering Method (QISM). The reader more interested in the physics of the model may choose to skip section 3. In section 4 , the ground state properties, the quantum phase diagram and the boundary impurity effects are studied via the TBA. A summary and discussion of our main results is given in section 5 .

\section{The integrable spin ladder model with boundary impurities}

Let us begin by introducing the integrable spin ladder model based on the $S U(4)$ symmetry with boundary fields,

$$
H=\frac{J_{\|}}{\gamma} H_{\operatorname{leg}}+J_{\perp} \sum_{j=1}^{L} \vec{S}_{j} \cdot \vec{T}_{j}+H_{1}^{(m)}+H_{L}^{(l)},
$$

where the leg part consists of Heisenberg exchange and four-spin interaction terms

$$
H_{\mathrm{leg}}=\sum_{j=1}^{L-1}\left(\frac{1}{4}+\vec{S}_{j} \cdot \vec{S}_{j+1}+\vec{T}_{j} \cdot \vec{T}_{j+1}+4 \vec{S}_{j} \cdot \vec{S}_{j+1} \cdot \vec{T}_{j} \cdot \vec{T}_{j+1}\right) .
$$

The left (right) boundary terms $H_{1}^{(m)}\left(H_{L}^{(l)}\right)$ depend on arbitrary parameters $U_{ \pm}$and are given by

$$
H_{1}^{(m)}= \begin{cases}-U_{-} \vec{S}_{1} \cdot \vec{T}_{1}-\frac{1}{4} U_{-}, & \text {for } m=1 \\ -U_{-}\left(\frac{1}{2}-S_{1}^{z}\right)\left(\frac{1}{2}-T_{1}^{z}\right)+\frac{1}{2} U_{-}, & \text {for } m=2 \\ U_{-}\left(\vec{S}_{1} \cdot \vec{T}_{1}-\left(\frac{1}{2}-S_{1}^{z}\right)\left(\frac{1}{2}-T_{1}^{z}\right)\right)+\frac{1}{4} U_{-}, & \text {for } m=3, \\ U_{-}\left(\vec{S}_{1} \cdot \vec{T}_{1}-\left(\frac{1}{2}+S_{1}^{z}\right)\left(\frac{1}{2}+T_{1}^{z}\right)\right)+\frac{1}{4} U_{-}, & \text {for } m=4 \\ -2 U_{-} S_{1}^{z} T_{1}^{z}+\frac{1}{2} U_{-}, & \text {for } m=5\end{cases}
$$




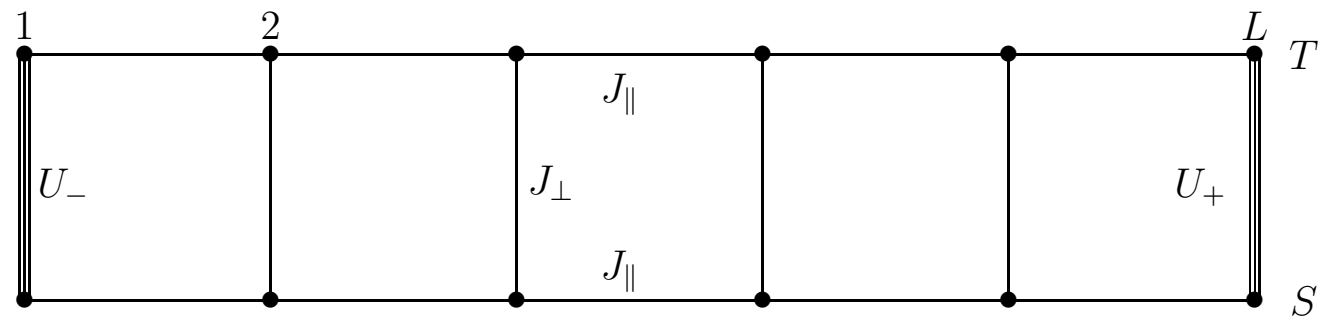

Fig. 1 . The $s u(4)$ spin ladder with boundary impurities. $U_{ \pm}$are the boundary impurity coupling constants. $J_{\|}$and $J_{\perp}$ are the intrachain and interchain couplings.

$$
H_{L}^{(l)}= \begin{cases}-U_{+} \vec{S}_{L} \cdot \vec{T}_{L}-\frac{1}{4} U_{+}, & \text {for } l=1 \\ -U_{+}\left(\frac{1}{2}-S_{L}^{z}\right)\left(\frac{1}{2}-T_{L}^{z}\right)+\frac{1}{2} U_{+}, & \text {for } l=2 \\ U_{+}\left(\vec{S}_{L} \cdot \vec{T}_{L}-\left(\frac{1}{2}-S_{L}^{z}\right)\left(\frac{1}{2}-T_{L}^{z}\right)\right)+\frac{1}{4} U_{+}, & \text {for } l=3 \\ U_{+}\left(\vec{S}_{L} \cdot \vec{T}_{L}-\left(\frac{1}{2}+S_{L}^{z}\right)\left(\frac{1}{2}+T_{L}^{z}\right)\right)+\frac{1}{4} U_{+}, & \text {for } l=4 \\ -2 U_{+} S_{L}^{z} T_{L}^{z}+\frac{1}{2} U_{+}, & \text {for } l=5\end{cases}
$$

In the above $\vec{S}_{j}$ and $\vec{T}_{j}$ are the standard spin- $\frac{1}{2}$ operators acting on site $j$ of the upper and lower legs, respectively, $J_{\|}$and $J_{\perp}$ are the intrachain and interchain couplings (see figure 1 ) and $L$ is the length of the ladder. It is worth noticing that $\gamma$ is a rescaling constant which can be used to minimize the biquadratic term such that the quantum phase of the model (1) lies in the same Haldane spin liquid phase as that of the conventional spin ladder (see section 4).

Notice that the boundary terms corresponding to the first case $(m=1, l=1)$ in (3) and (4) act as Heisenberg-type rung couplings, whereas in the second case $(m=2, l=2)$ they act as a $z$-component spin interaction with boundary magnetic fields. In the third $(m=3, l=3)$ and fourth $(m=4, l=4)$ cases, they act as a combination of Heisenberg-type rung coupling and $z$-component spin interaction with boundary magnetic fields. In the last case $(m=5, l=5)$ only the $z$-component spin interaction terms survive. The Hamiltonian (1) thus contains five different types of boundary rung interactions at each edge of the ladder realizing different impurity dopings. This leads to twenty five possible choices of boundary impurities. The rung interaction in the bulk was, as usual, introduced by the chemical potential terms given by $-J_{\perp} \sum_{j=1}^{L} e_{j}{ }^{11}$ in the canonical basis $e_{i} \otimes e_{j}$. The rung states split into a singlet and a triplet denoted by

$$
|1\rangle=\frac{1}{\sqrt{2}}(|\uparrow \downarrow\rangle-|\downarrow \uparrow\rangle),|2\rangle=|\uparrow \uparrow\rangle
$$




$$
|3\rangle=\frac{1}{\sqrt{2}}(|\uparrow \downarrow\rangle+|\downarrow \uparrow\rangle),|4\rangle=|\downarrow \downarrow\rangle,
$$

respectively. The leg interaction part of the Hamiltonian (2) does not change under the basis transformation (5). However, the bulk rung interaction part and the boundary rung interaction terms alter with respect to the choice of the order of singlet and triplet in the basis (5).

In order to derive this model let us begin by recalling the $S U(4) R$-matrix

$$
R_{12}(u)=\left(\begin{array}{cccccccccccccccc}
w_{1} & 0 & 0 & 0 & 0 & 0 & 0 & 0 & 0 & 0 & 0 & 0 & 0 & 0 & 0 & 0 \\
0 & w_{2} & 0 & 0 & w_{3} & 0 & 0 & 0 & 0 & 0 & 0 & 0 & 0 & 0 & 0 & 0 \\
0 & 0 & w_{2} & 0 & 0 & 0 & 0 & 0 & w_{3} & 0 & 0 & 0 & 0 & 0 & 0 & 0 \\
0 & 0 & 0 & w_{2} & 0 & 0 & 0 & 0 & 0 & 0 & 0 & 0 & w_{3} & 0 & 0 & 0 \\
0 & w_{3} & 0 & 0 & w_{2} & 0 & 0 & 0 & 0 & 0 & 0 & 0 & 0 & 0 & 0 & 0 \\
0 & 0 & 0 & 0 & 0 & w_{1} & 0 & 0 & 0 & 0 & 0 & 0 & 0 & 0 & 0 & 0 \\
0 & 0 & 0 & 0 & 0 & 0 & w_{2} & 0 & 0 & w_{3} & 0 & 0 & 0 & 0 & 0 & 0 \\
0 & 0 & 0 & 0 & 0 & 0 & 0 & w_{2} & 0 & 0 & 0 & 0 & 0 & w_{3} & 0 & 0 \\
0 & 0 & w_{3} & 0 & 0 & 0 & 0 & 0 & w_{2} & 0 & 0 & 0 & 0 & 0 & 0 & 0 \\
0 & 0 & 0 & 0 & 0 & 0 & w_{3} & 0 & 0 & w_{2} & 0 & 0 & & 0 & 0 & 0 \\
0 & 0 & 0 & 0 & 0 & 0 & 0 & 0 & 0 & 0 & w_{1} & 0 & 0 & 0 & 0 & 0 \\
0 & 0 & 0 & 0 & 0 & 0 & 0 & 0 & 0 & 0 & 0 & w_{2} & 0 & 0 & w_{3} & 0 \\
0 & 0 & 0 & w_{3} & 0 & 0 & 0 & 0 & 0 & 0 & 0 & 0 & w_{2} & 0 & 0 & 0 \\
0 & 0 & 0 & 0 & 0 & 0 & 0 & w_{3} & 0 & 0 & 0 & 0 & 0 & w_{2} & 0 & 0 \\
0 & 0 & 0 & 0 & 0 & 0 & 0 & 0 & 0 & 0 & 0 & w_{3} & 0 & 0 & w_{2} & 0 \\
0 & 0 & 0 & 0 & 0 & 0 & 0 & 0 & 0 & 0 & 0 & 0 & 0 & 0 & 0 & w_{1}
\end{array}\right)
$$

where the elements are given by

$$
w_{1}=u+1, \quad w_{2}=u, \quad w_{3}=1 .
$$

The quantum $R$-matrix (6) satisfies the Yang-Baxter equation (YBE)

$$
R_{12}(u-v) R_{13}(u) R_{23}(v)=R_{23}(v) R_{13}(u) R_{12}(u-v),
$$

guaranteeing the integrability of the model with periodic BC. This $R$-matrix enjoys the properties

$$
R_{12}(u) R_{21}(-u)=1-u^{2}, \quad R_{12}^{\mathrm{t}_{1} \mathrm{t}_{2}}(u)=R_{12}(u),
$$

where superscript $\mathrm{t}_{a}$ denotes the transposition in the space with index $a$. For other types of boundary conditions, the YBE will still account for the integrability of the bulk part of the model, but the boundary terms have to be chosen appropriately in order to preserve the integrability. In particular, 
the left and right reflection matrices, $K_{-}$and $K_{+}$, respectively, are required to satisfy the RE's $[17,19]$

$$
\begin{aligned}
& R_{12}(u-v) \stackrel{1}{K}-(u) R_{21}(u+v) \stackrel{2}{K_{-}}(v) \\
& =\stackrel{2}{K}(v) R_{12}(u+v) \stackrel{1}{K}(u) R_{21}(u-v), \\
& R_{21}^{\mathrm{t}_{1} \mathrm{t}_{2}}(v-u) \stackrel{1}{K_{+}^{\mathrm{t}_{1}}}(u) \tilde{R}_{12}(-u-v) \stackrel{2}{\mathrm{t}_{2}}(v) \\
& \stackrel{2}{\mathrm{t}_{+}^{\mathrm{t}_{2}}}(v) R_{21}^{\mathrm{t}_{1} \mathrm{t}_{2}}(-u-v) \stackrel{1}{K_{+}^{\mathrm{t}_{1}}}(u) R_{12}^{\mathrm{t}_{1} \mathrm{t}_{2}}(v-u) .
\end{aligned}
$$

In the above we have introduced the object $\tilde{R}$ which may be determined by the relations

$$
\tilde{R}_{12}^{\mathrm{t}_{2}}(-u) R_{21}^{\mathrm{t}_{1}}(u)=1, \quad \tilde{R}_{21}^{\mathrm{t}_{1}}(-u) R_{12}^{\mathrm{t}_{2}}(u)=1,
$$

and we have used the conventional notation

$$
\stackrel{1}{X} \equiv X \otimes \mathbf{I}_{V_{2}}, \quad \stackrel{2}{X} \equiv \mathbf{I}_{V_{1}} \otimes X,
$$

where $\mathbf{I}_{V}$ denotes the identity operator on $V$ and, as usual, $R_{21}=\mathbf{P} \cdot R_{12} \cdot \mathbf{P}$, with $\mathbf{P}$ being the permutation operator. After a lengthy calculation we find the possible solutions of the RE's for the diagonal $K_{ \pm}$-matrices (see also Ref. [18])

$$
K_{ \pm}(u)=\left(\begin{array}{cccc}
K 1_{ \pm}(u) & 0 & 0 & 0 \\
0 & K 2_{ \pm}(u) & 0 & 0 \\
0 & 0 & K 3_{ \pm}(u) & 0 \\
0 & 0 & 0 & K 4_{ \pm}(u)
\end{array}\right)
$$

The solutions for $K_{-}$, corresponding to the left boundary are:

Case 1

$$
K 1_{-}(u)=u+\xi_{-}, K 2_{-}(u)=K 3_{-}(u)=K 4_{-}(u)=-u+\xi_{-} ;
$$

Case 2

$$
K 1_{-}(u)=K 2_{-}(u)=K 3_{-}(u)=u+\xi_{-}, K 4_{-}(u)=-u+\xi_{-} ;
$$

Case 3

$$
K 1_{-}(u)=K 4_{-}(u)=-u+\xi_{-}, K 2_{-}(u)=K 3_{-}(u)=u+\xi_{-} ;
$$


Case 4

$$
K 1_{-}(u)=K 2_{-}(u)=-u+\xi_{-}, K 3_{-}(u)=K 4_{-}(u)=u+\xi_{-} ;
$$

Case 5

$$
K 1_{-}(u)=K 3_{-}(u)=u+\xi_{-}, K 2_{-}(u)=K 4_{-}(u)=-u+\xi_{-} .
$$

On the other hand, the solutions for $K_{+}$, corresponding to the right boundary, are:

Case 1

$$
\begin{aligned}
& K 1_{+}(u)=-u+\xi_{+}+3 \\
& K 2_{+}(u)=K 3_{+}(u)=K 4_{+}(u)=u-\xi_{+}+1
\end{aligned}
$$

Case 2

$$
\begin{aligned}
& K 1_{+}(u)=K 2_{+}(u)=K 3_{+}(u)=-u-\xi_{+}-1 \\
& K 4_{+}(u)=u-\xi_{+}+3
\end{aligned}
$$

Case 3

$$
\begin{aligned}
& K 1_{+}(u)=K 4_{+}(u)=u-\xi_{+}+2 \\
& K 2_{+}(u)=K 3_{+}(u)=-u-\xi_{+}-2
\end{aligned}
$$

Case 4

$$
\begin{aligned}
& K 1_{+}(u)=K 2_{+}(u)=u-\xi_{+}+2 \\
& K 3_{+}(u)=K 4_{+}(u)=-u-\xi_{+}-2
\end{aligned}
$$

Case 5

$$
\begin{aligned}
& K 1_{+}(u)=K 3_{+}(u)=-u-\xi_{+}-2 \\
& K 2_{+}(u)=K 4_{+}(u)=u-\xi_{+}+2
\end{aligned}
$$

In the above $\xi_{ \pm}=\frac{J_{\|}}{\gamma U_{ \pm}}$are free parameters related to the left $-($right +$)$ boundary coupling $U_{-}\left(U_{+}\right)$respectively. The boundary pairs $K_{-}^{(m)}(u), K_{+}^{(l)}(u)$, $l, m=1, \ldots, 5$, lead to the boundary pair terms $H_{-}^{(m)}$ and $H_{+}^{(l)}$ in $(3)$ and $(4)$, 
whose combination leads to twenty five possible choices of boundary impurities. A special choice of the boundary term $H^{(1)}$ was already investigated in [15]. The symmetries enjoyed by the $R$-matrix (6) and the RE's (10) and (11) constitute the necessary ingredients for the integrability of the model with boundary impurities, due to the fact that the double-row transfer matrix of the system

$$
\tau(u)=\operatorname{tr}_{0}\left[K_{+}(u) T(u) K_{-}(u) T^{-1}(-u)\right]
$$

commutes for different values of the spectral parameter. Here $T(u)$ denotes the monodromy matrix given by

$$
T(u)=R_{0, L}(u) R_{0, L-1}(u) \cdots R_{0,2}(u) R_{0,1}(u)
$$

and $T^{-1}$ its inverse. The Hamiltonian (1) associated with the quantum $R$ matrix (6) is related to the double-row transfer matrix (25) by

$$
H=-\left.\frac{J_{\|}}{2 \gamma} \frac{d}{d u} \ln \tau(u)\right|_{u=0}-J_{\perp} \sum_{j=1}^{L} e_{j}^{11}+\text { const. }
$$

Here

$$
\left.\frac{d}{d u} \ln \tau(u)\right|_{u=0}=2 \sum_{j=1}^{L-1} H_{j j+1}+K_{-}^{-1}(0) K_{-}^{\prime}(0)+2 \frac{\operatorname{tr}_{0} K_{+}(0) R_{0 L}^{\prime}(0) P_{0 L}}{\operatorname{tr}_{0} K_{+}(0)}
$$

where the prime denotes the derivative with respect to the spectral parameter. The relation (27) clearly indicates the identification $U_{ \pm}=\frac{J_{\|}}{\gamma \xi_{ \pm}}$between the boundary impurity couplings $U_{ \pm}$and the free parameters $\xi_{ \pm}$of the boundary scattering matrices. So far, we have completed the first step towards the solution of the model with boundary impurities. Next we proceed with the diagonalization of the transfer matrix (25) by means of the open algebraic Bethe ansatz [24,25].

\section{The algebraic Bethe-ansatz approach}

\subsection{First-level nesting structure}

In order to find the spectrum of our Hamiltonian with boundary defects, we first need to solve the eigenvalue problem of the transfer matrix, namely 
$\tau \Phi=\lambda \Phi$. As usual, the transfer matrix (25) can be written in the form

$$
\tau(\lambda)=\operatorname{tr}_{0}\left[K_{+}(u) \tilde{T}_{-}(u)\right],
$$

where $\tilde{T}_{-}(u)$ is the double-row monodromy matrix defined by

$$
\tilde{T}_{-}(u)=T(u) K_{-}(u) T^{-1}(-u) .
$$

One can verify that $\tilde{T}_{-}(u)$ also satisfies the RE (10). Following the notation used in Refs. $[22,23,24,25]$, we label the elements of the monodromy matrix $T(u)$ by

$$
T(u)=\left(\begin{array}{cccc}
A(u) & B_{1}(u) & B_{2}(u) & B_{3}(u) \\
C_{1}(u) & D_{11}(u) & D_{12}(u) & D_{13}(u) \\
C_{2}(u) & D_{21}(u) & D_{22}(u) & D_{23}(u) \\
C_{3}(u) & D_{31}(u) & D_{32}(u) & D_{33}(u)
\end{array}\right),
$$

and further

$$
\begin{array}{r}
T^{-1}(-u)=\left(\begin{array}{cccc}
\bar{A}(u) & \bar{B}_{1}(u) & \bar{B}_{2}(u) & \bar{B}_{3}(u) \\
\bar{C}_{1}(u) & \bar{D}_{11}(u) & \bar{D}_{12}(u) & \bar{D}_{13}(u) \\
\bar{C}_{2}(u) & \bar{D}_{21}(u) & \bar{D}_{22}(u) & \bar{D}_{23}(u) \\
\bar{C}_{3}(u) & \bar{D}_{31}(u) & \bar{D}_{32}(u) & \bar{D}_{33}(u)
\end{array}\right), \\
\tilde{T}_{-}(u)=\left(\begin{array}{cccc}
\tilde{B}(u) & \tilde{A}_{1}(u) & \tilde{B}_{2}(u) & \tilde{B}_{3}(u) \\
\tilde{C}_{1}(u) & \tilde{D}_{11}(u) & \tilde{D}_{12}(u) & \tilde{D}_{13}(u) \\
\tilde{C}_{2}(u) & \tilde{D}_{21}(u) & \tilde{D}_{22}(u) & \tilde{D}_{23}(u) \\
\tilde{C}_{3}(u) & \tilde{D}_{31}(u) & \tilde{D}_{32}(u) & \tilde{D}_{33}(u)
\end{array}\right) .
\end{array}
$$

According to the first level Bethe ansatz, the eigenvectors $|\Phi\rangle$ of the transfer matrix can be written as

$$
|\Phi\rangle=\tilde{B}_{i_{1}}\left(u_{1}\right) \cdots \tilde{B}_{i_{N}}\left(u_{N}\right)|\phi\rangle F_{(1)}^{i_{1} \cdots i_{N}},
$$

where the summation is taken on the repeated indicies. The coefficients with indices $i_{n}=1,2,3, n=1, \cdots, N$ will be determined later by the second level Bethe ansatz. The first level pseudovacuum $|\phi\rangle$ is chosen as the standard ferromagnetic state

$$
|\phi\rangle=|0\rangle_{L} \otimes \cdots \otimes|0\rangle_{i} \otimes \cdots \otimes|0\rangle_{1},
$$

where $|0\rangle_{i}=(1,0,0,0)_{i}^{\mathrm{t}}$ acts as a highest-weight vector. This state corresponds to the product of the rung singlet state in the basis (5). Different choices 
of the order of the basis (5) will change the eigenvalues of the Hamiltonian which facilitates the analysis of the ground state in different regions. From the structure of the $R$-matrix (6), one can deduce that the operators $B_{i}(u)$ and $\bar{B}_{i}(u)(i=1,2,3)$ act on the reference state as creation operators creating particles with pseudo-momenta $u$ and $-u$, respectively. The operators $C_{i}(u)$ $(i=1, \cdots, 3)$ behave as annihilation fields. Furthermore, using an invariant version of the Yang-Baxter algebra,

$$
\stackrel{2}{T}^{-1}(-u) R_{12}(2 u) \stackrel{1}{T}(u)=\stackrel{1}{T}(u) R_{12}(2 u) \stackrel{2}{T}^{-1}(-u),
$$

we obtain, apart from an overall factor $Q(u)=K 1_{-}(u) K 1_{+}(u)$, the eigenvalue of the transfer matrix acting on the reference state $|\phi\rangle$

$$
\tau(u)|\phi\rangle=\left\{\omega_{A}^{+}(u) \tilde{A}(u)+\sum_{a=1}^{3} \omega_{a}^{+}(u) \hat{D}_{a a}(u)\right\}|\phi\rangle,
$$

where we have introduced the transformations

$$
\hat{D}_{i j}(u)=\tilde{D}_{i j}(u)-\delta_{i j} \frac{w_{3}(2 u)}{w_{1}(2 u)} \tilde{A}(u)=\omega_{i}^{-}(u) D_{i i}(u) \bar{D}_{i i}(u),
$$

with $i=1,2,3$ and

$$
\tilde{A}(u)=\omega_{A}^{-}(u) A(u) \bar{A}(u) .
$$

In the above expression,

$$
\begin{aligned}
& \omega_{A}^{-}(u)=1, \text { for } m=1, \ldots, 5, \\
& \omega_{A}^{+}(u)= \begin{cases}\frac{(u+2)\left(u+\xi_{+}\right)}{(u+1 / 2)\left(u+\xi_{+}+3\right)}, & \text { for } l=1, \\
\frac{(u+2)\left(u+\xi_{+}\right)}{(u+1 / 2)\left(u+\xi_{+}+1\right)}, & \text { for } l=2, \\
\frac{(u+2)\left(-u+\xi_{+}\right)}{(u+1 / 2)\left(-u+\xi_{+}-2\right)}, \text { for } l=3,4, & \frac{(u+2)\left(u+\xi_{+}\right)}{(u+1 / 2)\left(u+\xi_{+}+2\right)}, \text { for } l=5,\end{cases} \\
& \omega_{1}^{-}(u)= \begin{cases}\frac{u\left(-u+\xi_{-}-1\right)}{(u+1 / 2)\left(u+\xi_{-}\right)}, & \text {for } m=1, \\
\frac{u\left(u+\xi_{-}+1\right)}{(u+1 / 2)\left(-u+\xi_{-}\right)}, & \text {for } m=3, \\
\frac{u}{u+1 / 2}, \quad \text { for } m=2,4, \\
\frac{u\left(-u+\xi_{-}-1\right)}{(u+1 / 2)\left(u+\xi_{-}\right)}, & \text {for } m=5,\end{cases}
\end{aligned}
$$




$$
\begin{aligned}
& \omega_{2}^{-}(u)= \begin{cases}\frac{u\left(-u+\xi_{-}-1\right)}{(u+1 / 2)\left(u+\xi_{-}\right)}, & \text {for } m=1, \\
\frac{u\left(u+\xi_{-}+1\right)}{(u+1 / 2)\left(-u+\xi_{-}\right)}, & \text {for } m=3, \\
\frac{u\left(u+\xi_{-}+1\right)}{(u+1 / 2)\left(-u+\xi_{-}\right)}, & \text {for } m=4, \\
\frac{u}{u+1 / 2}, & \text { for } m=2,5,\end{cases} \\
& \omega_{3}^{-}(u)= \begin{cases}\frac{u\left(-u+\xi_{-}-1\right)}{(u+1 / 2)\left(u+\xi_{-}\right)}, & \text {for } m=1, \\
\frac{u}{u+1 / 2}, & \text { for } m=3, \\
\frac{u\left(u+\xi_{-}+1\right)}{(u+1 / 2)\left(-u+\xi_{-}\right)}, & \text {for } m=4, \\
\frac{u\left(-u+\xi_{-}-1\right)}{(u+1 / 2)\left(u+\xi_{-}\right)}, & \text {for } m=2,5,\end{cases}
\end{aligned}
$$

with $\omega_{1}^{+}(u)=K 2_{+}(u), \omega_{2}^{+}(u)=K 3_{+}(u), \omega_{3}^{+}(u)=K 4_{+}(u)$ for $l=1, \ldots, 5$. In addition,

$$
A(u) \bar{A}(u)|0\rangle=w_{1}(u)^{2}|0\rangle, \quad D_{i i}(u) \bar{D}_{i i}(u)|0\rangle=w_{2}(u)^{2}|0\rangle .
$$

We note that the operators $\tilde{B}_{i}(u), i=1,2,3$, constitute a three-component vector with both positive and negative pseudo-momenta still playing the role of the creation fields acting on the pseudovacuum state. In order to make further progress we return to the $\mathrm{RE}(10)$ and derive the commutation relations

$$
\begin{aligned}
& \tilde{A}\left(u_{1}\right) \tilde{B}_{a}\left(u_{2}\right)=\frac{\left(u_{2}-u_{1}+1\right)\left(u_{1}+u_{2}\right)}{\left(u_{2}-u_{1}\right)\left(u_{1}+u_{2}+1\right)} \tilde{B}_{a}\left(u_{2}\right) \tilde{A}\left(u_{1}\right) \\
& -\frac{\left(u_{1}+u_{2}\right)}{\left(u_{2}-u_{1}\right)\left(u_{1}+u_{2}+1\right)} \tilde{B}_{a}\left(u_{1}\right) \tilde{A}\left(u_{2}\right) \\
& -\frac{1}{u_{1}+u_{2}+1}\left[\sum_{b=1}^{3} \tilde{B}_{b}\left(u_{1}\right) \hat{D}_{b a}\left(u_{2}\right)+\delta_{a b} \frac{1}{2 u_{2}+1} \tilde{B}_{b}\left(u_{1}\right) A\left(u_{2}\right)\right], \\
& \hat{D}_{b d}\left(u_{1}\right) \tilde{B}_{c}\left(u_{2}\right)=\frac{\left(u_{1}-u_{2}+1\right)\left(u_{1}+u_{2}+2\right)}{\left(u_{1}-u_{2}\right)\left(u_{1}+u_{2}+1\right)} \\
& \times\left\{r^{(1)}\left(u_{1}+u_{2}+1\right)_{g h}^{e b} r^{(1)}\left(u_{1}-u_{2}\right)_{c d}^{i h} \tilde{B}_{e}\left(u_{2}\right) \hat{D}_{g i}\left(u_{1}\right)\right\} \\
& -\frac{2\left(u_{1}+1\right)}{\left(2 u_{1}+1\right)\left(u_{1}-u_{2}\right)} r^{(1)}\left(2 u_{1}+1\right)_{i d}^{g b} \tilde{B}_{g}\left(u_{1}\right) \hat{D}_{i c}\left(u_{2}\right) \\
& +\frac{4\left(u_{1}+1\right) u_{2}}{\left(2 u_{1}+1\right)\left(2 u_{2}+1\right)\left(u_{1}+u_{2}+1\right)} r^{(1)}\left(2 u_{1}+1\right)_{c d}^{g b} \tilde{B}_{g}\left(u_{1}\right) \tilde{A}\left(u_{2}\right),
\end{aligned}
$$

between the diagonal fields and the creation fields. The summation convention is implied for repeated indices. The matrix $r^{(1)}$, which satisfies the Yang-Baxter equation, takes the form

$$
r_{a a}^{a a}=1, a=1,2,3, \quad r_{a b}^{a b}=\frac{1}{u+1}, a \neq b=1,2,3
$$




$$
r_{b a}^{a b}=\frac{u}{u+1}, a \neq b=1,2,3
$$

We notice that the first term in the rhs of each of the commutation relations (40)-(41) contribute to the eigenvalues of the transfer matrix, which should be analytic functions of the spectral parameter $u$. Consequently, the residues at singular points must vanish. This yields the Bethe ansatz equations, which in turn assure the cancellation of the unwanted terms in the eigenvalues of the transfer matrix after the whole nesting procedure. For convenience, we make a shift in the spectral parameters, $u=v-1 / 2, u_{i}=v_{i}-1 / 2$, such that the eigenvalue of the transfer matrix (25) can be obtained as

$$
\begin{aligned}
& \tau(v)|\Phi\rangle=\Lambda\left(v,\left\{v_{i}\right\}\right)|\Phi\rangle \\
& =W_{A}^{+}(v-1 / 2) W_{A}^{-}(v-1 / 2) \prod_{i=1}^{N} \frac{\left(v-v_{i}-1\right)\left(v+v_{i}-1\right)}{\left(v-v_{i}\right)\left(v+v_{i}\right)}|\Phi\rangle \\
& +W_{a}^{+}(v-1 / 2) W_{a}^{-}(v-1 / 2) \prod_{i=1}^{N} \frac{\left(v-v_{i}+1\right)\left(v+v_{i}+1\right)}{\left(v-v_{i}\right)\left(v+v_{i}\right)} \Lambda^{(1)}\left(v,\left\{v_{i}\right\}\right)|\Phi\rangle,
\end{aligned}
$$

provided that

$$
\begin{array}{r}
\frac{W_{A}^{+}\left(v_{i}-1 / 2\right) W_{A}^{-}\left(v_{i}-1 / 2\right)\left(2 v_{i}-1\right)}{W_{1}^{+}\left(v_{i}-1 / 2\right) W_{1}^{-}\left(v_{i}-1 / 2\right)\left(2 v_{i}+1\right)} \\
=\left.\prod_{\substack{l=1 \\
l \neq i}}^{N} \frac{\left(v_{i}-v_{l}+1\right)\left(v_{i}+v_{l}+1\right)}{\left(v_{i}-v_{l}-1\right)\left(v_{i}+v_{l}-1\right)} \Lambda^{(1)}\left(v,\left\{v_{i}\right\}\right)\right|_{v=v_{i}} .
\end{array}
$$

Here $a=1,2,3$, and we appropriately choose

$$
\begin{gathered}
W_{A}^{-}(u)=1, \text { for } m=1, \ldots, 5, \\
W_{A}^{+}(u)= \begin{cases}\frac{(u+2)\left(u+\xi_{+}\right)}{(u+1 / 2)\left(u+\xi_{+}+3\right)}, & \text { for } l=1, \\
\frac{(u+2)\left(u+\xi_{+}\right)}{(u+1 / 2)\left(u+\xi_{+}+1\right)}, & \text { for } l=2, \\
\frac{(u+2)\left(-u+\xi_{+}\right)}{(u+1 / 2)\left(-u+\xi_{+}-2\right)}, \text { for } l=3,4, & \frac{(u+2)\left(u+\xi_{+}\right)}{(u+1 / 2)\left(u+\xi_{+}+2\right)}, \text { for } l=5,\end{cases} \\
W_{a}^{+}(u)= \begin{cases}\frac{u-\xi_{+}+1}{-u-\xi_{+}-3}, \text { for } l=1, \\
1, \quad \text { for } l=2, \ldots, 5,\end{cases} \\
W_{a}^{-}(u)= \begin{cases}\frac{u\left(-u+\xi_{-}-1\right)}{(u+1 / 2)\left(u+\xi_{-}\right)}, \text {for } m=1, \\
\frac{u}{u+1 / 2}, \quad \text { for } m=2, \ldots, 5 .\end{cases}
\end{gathered}
$$


$\Lambda^{(1)}\left(v,\left\{v_{i}\right\}\right)$ is the eigenvalue of the second level transfer matrix $\tau^{(1)}$ related to an $S U(3)$ invariant open chain, i.e.

$$
\tau^{(1)}=\operatorname{Tr}_{0} K_{+}^{(1)}(v) T^{(1)}(v) K_{-}^{(1)}(v) \bar{T}^{(1)}(v)
$$

where

$$
\begin{aligned}
& T^{(1)}\left(v,\left\{v_{i}\right\}\right)=r_{12}^{(1)}\left(v+v_{1}\right)_{h_{1} g_{1}}^{e_{1} a} \ldots r_{12}^{(1)}\left(v+v_{N}\right)_{h_{N} g_{N}}^{e_{N} g_{N-1}} \\
& \bar{T}^{(1)}\left(v,\left\{v_{i}\right\}\right)=r_{21}^{(1)}\left(v-v_{N}\right)_{l_{N-1} e_{N} h_{N}}^{e_{N}} \ldots r_{21}^{(1)}\left(v-v_{1}\right)_{a i_{1}}^{e_{1} h_{1}}
\end{aligned}
$$

Here we have used the standard notation $r_{12}^{(1)}(v)=P \cdot r^{(1)}(v)$ where $P$ is the standard permutation operator, which can be represented by a $3^{2} \times 3^{2}$ matrix, i.e., $p_{\alpha \beta, \gamma \delta}=\delta_{\alpha \delta} \delta_{\beta \gamma}$. It can be seen that the coefficients $F_{(1)}^{i_{1}, \ldots, i_{n}}$ act as the multi-particle vectors for the inhomogeneous transfer matrix (49). We remark that the coefficients $W$ given in (45)-(48) are chosen in order to match the choice of the transfer matrix (49) with the nested $K_{ \pm}^{(1)}$-matrices,

$$
K_{ \pm}^{(1)}(v)=\left(\begin{array}{ccc}
K 1_{ \pm}^{(1)}(v) & 0 & 0 \\
0 & K 2_{ \pm}^{(1)}(v) & 0 \\
0 & 0 & K 3_{ \pm}^{(1)}(v)
\end{array}\right)
$$

Now corresponding to the first solution (20), we have

$$
\begin{aligned}
& K 1_{-}^{(1)}(v)=K 2_{-}^{(1)}(v)=K 3_{-}^{(1)}(v)=1 \\
& K 1_{+}^{(1)}(v)=K 2_{+}^{(1)}(v)=K 3_{+}^{(1)}(v)=1
\end{aligned}
$$

And to the second solution (21):

$$
\begin{aligned}
& K 1_{-}^{(1)}(v)=K 2_{-}^{(1)}(v)=1, \quad K 3_{-}^{(1)}(v)=\frac{-v+\xi_{-}-1 / 2}{v+\xi_{-}-1 / 2} \\
& K 1_{+}^{(1)}(v)=K 2_{+}^{(1)}(v)=1, \quad K 3_{+}^{(1)}(v)=\frac{v-\xi_{+}+5 / 2}{-v-\xi_{+}-1 / 2} .
\end{aligned}
$$

And to the third solution (22):

$$
\begin{aligned}
& K 1_{-}^{(1)}(v)=K 2_{-}^{(1)}(v)=\frac{v+\xi_{-}+1 / 2}{-v+\xi_{-}+1 / 2}, \quad K 3_{-}^{(1)}(v)=1 \\
& K 1_{+}^{(1)}(v)=K 2_{+}^{(1)}(v)=\frac{-v-\xi_{+}-3 / 2}{v-\xi_{+}+3 / 2}, \quad K 3_{+}^{(1)}(v)=1 .
\end{aligned}
$$


And to the fourth solution (23):

$$
\begin{aligned}
& K 1_{-}^{(1)}(v)=1, K 2_{-}^{(1)}(v)=K 3_{-}^{(1)}(v)=\frac{v+\xi_{-}+1 / 2}{-v+\xi_{-}+1 / 2}, \\
& K 1_{+}^{(1)}(v)=1, K 2_{+}^{(1)}(v)=K 3_{+}^{(1)}(v)=\frac{-v-\xi_{+}-3 / 2}{v-\xi_{+}+3 / 2} .
\end{aligned}
$$

And to the last solution (24):

$$
\begin{aligned}
& K 2_{-}^{(1)}(v)=1, \quad K 1_{-}^{(1)}(v)=K 3_{-}^{(1)}(v)=\frac{-v+\xi_{-}-1 / 2}{v+\xi_{-}-1 / 2} \\
& K 2_{+}^{(1)}(v)=1, \quad K 1_{+}^{(1)}(v)=K 3_{+}^{(1)}(v)=\frac{v-\xi_{+}+3 / 2}{-v-\xi_{+}-3 / 2} .
\end{aligned}
$$

We can show that the reflection matrices (52) with the entries (53)-(62) do satisfy the RE

$$
\begin{aligned}
& r_{12}^{(1)}(u-v) \stackrel{1}{K_{-}^{(1)}}(u) r_{21}^{(1)}(u+v) \stackrel{2}{K_{-}^{(1)}}(v) \\
& =K^{(1)}-(v) r_{12}^{(1)}(u+v) K_{-}^{(1)}(u) r_{21}^{(1)}(u-v), \\
& r_{21}^{(1)^{\mathrm{t}_{1} \mathrm{t}_{2}}}(v-u) K_{+}^{1^{(1)}}(u) \tilde{r}_{12}^{(1)}(-u-v) K_{+}^{\mathrm{t}_{1}}(v) \\
& =K_{+}^{2^{(1)}}(v) r_{21}^{(1)^{\mathrm{t}_{2} \mathrm{t}_{2}}}(-u-v) K_{+}^{(1)}(u) r_{12}^{(1)^{\mathrm{t}_{1} \mathrm{t}_{2}}}(v-u) .
\end{aligned}
$$

\subsection{Second-level Bethe ansatz}

In order to proceed in the nested algebraic Bethe ansatz, we have to repeat the whole procedure presented above for the internal block of the monodromy matrix. Similarly, we rewrite the second level transfer matrix $\tau^{(1)}$ (49) in the form

$$
\tau^{(1)}(v)=\operatorname{Tr}_{0}\left[K_{+}^{(1)}(v) U_{-}^{(1)}(v)\right]
$$

where $U_{-}^{(1)}(v)$ is defined by

$$
\tilde{T}_{-}^{(1)}(v)=T^{(1)}(v) K_{-}^{(1)}(v) \bar{T}^{(1)}(v) .
$$

Now we label the elements of the monodromy matrices by 


$$
\begin{aligned}
T^{(1)}(\lambda) & =\left(\begin{array}{lll}
A^{(1)}(v) & B_{1}^{(1)}(v) & B_{2}^{(1)}(v) \\
C_{1}^{(1)}(v) & D_{11}^{(1)}(v) & D_{12}^{(1)}(v) \\
C_{2}^{(1)}(v) & D_{21}^{(1)}(v) & D_{22}^{(1)}(v)
\end{array}\right), \\
\bar{T}^{(1)}(v) & =\left(\begin{array}{lll}
\bar{A}^{(1)}(v) & \bar{B}_{1}^{(1)}(v) & \bar{B}_{2}^{(1)}(v) \\
\bar{C}_{1}^{(1)}(v) & \bar{D}_{11}^{(1)}(v) & \bar{D}_{12}^{(1)}(v) \\
\bar{C}_{2}^{(1)}(v) & \bar{D}_{21}^{(1)}(v) & \bar{D}_{22}^{(1)}(v)
\end{array}\right), \\
\tilde{T}_{-}^{(1)}(v) & =\left(\begin{array}{lll}
\tilde{A}^{(1)}(v) & \tilde{B}_{1}^{(1)}(v) & \tilde{B}_{2}^{(1)}(v) \\
\tilde{C}_{1}^{(1)}(v) & \tilde{D}_{11}^{(1)}(v) & \tilde{D}_{12}^{(1)}(v) \\
\tilde{C}_{2}^{(1)}(v) & \tilde{D}_{21}^{(1)}(v) & \tilde{D}_{22}^{(1)}(v)
\end{array}\right) .
\end{aligned}
$$

From the structure of the $r^{(1)}$-matrix (42), it is found that the operators $B_{a}^{(1)}(v)$ and $\bar{B}_{a}^{(1)}(v)(a=1,2)$ act as creation fields acting on the reference state $|0\rangle_{i}=\left(\begin{array}{l}1 \\ 0 \\ 0\end{array}\right)_{i}$. The operators $C_{i}^{(1)}(v)(i=1, \cdots, 2)$ behave as annihilation fields. In order to make further progress we return to the RE (63) and derive commutation relations,

$$
\begin{aligned}
& \tilde{A}^{(1)}\left(v_{1}\right) \tilde{B}_{a}^{(1)}\left(v_{2}\right)=\frac{\left(v_{1}-v_{2}-1\right)\left(v_{1}+v_{2}\right)}{\left(v_{1}-v_{2}\right)\left(v_{1}+v_{2}+1\right)} \tilde{B}_{a}^{(1)}\left(v_{2}\right) \tilde{A}^{(1)}\left(v_{1}\right) \\
& +\frac{\left(v_{1}+v_{2}\right)}{\left(v_{1}-v_{2}\right)\left(v_{1}+v_{2}+1\right)} \tilde{B}_{a}^{(1)}\left(v_{1}\right) \tilde{A}^{(1)}\left(v_{2}\right) \\
& -\frac{1}{v_{1}+v_{2}+1}\left[\sum_{b=1}^{2} \tilde{B}_{b}^{(1)}\left(v_{1}\right) \hat{D}_{b a}^{(1)}\left(v_{2}\right)+\delta_{a b} \frac{1}{2 v_{2}+1} \tilde{B}_{b}^{(1)}\left(v_{1}\right) A^{(1)}\left(v_{2}\right)\right], \\
& \hat{D}_{b d}^{(1)}\left(v_{1}\right) \tilde{B}_{c}^{(1)}\left(v_{2}\right)=\frac{\left(v_{1}-v_{2}+1\right)\left(v_{1}+v_{2}+2\right)}{\left(v_{1}+v_{2}+1\right)\left(v_{1}-v_{2}\right)} \\
& \times\left\{r^{(2)}\left(v_{1}+v_{2}+1\right)_{g h}^{e b} r^{(2)}\left(v_{1}-v_{2}\right)_{c d}^{i h} \tilde{B}_{e}^{(1)}\left(v_{2}\right) \hat{D}_{g i}\left(v_{1}\right)\right\} \\
& -\frac{2\left(v_{1}+1\right)}{\left(2 v_{1}+1\right)\left(v_{1}-v_{2}\right)} r^{(2)}\left(2 v_{1}+1\right)_{i d}^{g b} \tilde{B}_{g}^{(1)}\left(u_{1}\right) \hat{D}_{i c}^{(1)}\left(v_{2}\right) \\
& +\frac{4\left(v_{1}+1\right) v_{2}}{\left(2 v_{1}+1\right)\left(2 v_{2}+1\right)\left(v_{1}+v_{2}+1\right)} r^{(1)}\left(2 v_{1}+1\right)_{c d}^{g b} \tilde{B}_{g}^{(1)}\left(v_{1}\right) \tilde{A}^{(1)}\left(v_{2}\right),
\end{aligned}
$$

between the diagonal and the creation fields. Where again the summation convention is implied for the repeated indices. The matrix $r^{(2)}(v)$ is nothing but the $S U(2)$ invariant $R$-matrix, i.e.

$$
\begin{aligned}
& r_{a a}^{a a}=1, a=1,2, \quad r_{a b}^{a b}=\frac{1}{v+1}, a \neq b=1,2, \\
& r_{b a}^{a b}=\frac{v}{v+1}, a \neq b=1,2 .
\end{aligned}
$$


If we define the second level Bethe ansatz as

$$
\left|\Psi^{(1)}\right\rangle=\tilde{B}_{l_{1}}^{(1)}\left(\mu_{1}\right) \cdots \tilde{B}_{l_{M}}^{(1)}\left(\mu_{M}\right)|0\rangle F_{(2)}{ }^{l_{1} \cdots l_{M}},
$$

and make the rescalings $\mu_{l} \rightarrow \mu_{l}-1 / 2$ and $v=\tilde{v}-1 / 2$ we obtain from the commutation relations (70) and (71) the eigenvalue $\Lambda^{(1)}\left(v,\left\{v_{i}\right\}\left\{\mu_{j}\right\}\right)$, i.e.

$$
\begin{aligned}
& \tau^{(1)}(\tilde{v})\left|\Psi^{(1)}\right\rangle=\Lambda^{(1)}\left(\tilde{v},\left\{v_{i}\right\}\left\{\mu_{j}\right\}\right)\left|\Psi^{(1)}\right\rangle \\
& =\left\{W_{+A}^{(1)}(\tilde{v}-1 / 2) W_{-A}^{(1)}(\tilde{v}-1 / 2) \prod_{l=1}^{M} \frac{\left(\tilde{v}-\mu_{l}-1\right)\left(\tilde{v}+\mu_{l}-1\right)}{\left(\tilde{v}-\mu_{l}\right)\left(\tilde{v}+\mu_{l}\right)}\right. \\
& +W_{+a}^{(1)}(\tilde{v}-1 / 2) W_{-a}^{(1)}(\tilde{v}-1 / 2) \prod_{i=1}^{N} \frac{\left(\tilde{v}-v_{i}-1 / 2\right)\left(\tilde{v}+v_{i}-1 / 2\right)}{\left(\tilde{v}-v_{i}+1 / 2\right)\left(\tilde{v}+v_{i}+1 / 2\right)} \\
& \left.\times \prod_{l=1}^{M} \frac{\left(\tilde{v}-\mu_{l}+1\right)\left(\tilde{v}+\mu_{l}+1\right)}{\left(\tilde{v}-\mu_{l}\right)\left(\tilde{v}+\mu_{l}\right)} \Lambda^{(2)}\left(\tilde{v},\left\{v_{i}\right\},\left\{\mu_{l}\right\}\right)\right\}\left|\Phi^{(1)}\right\rangle,
\end{aligned}
$$

provided that

$$
\begin{aligned}
& \frac{W_{+A}^{(1)}\left(\mu_{l}-1 / 2\right) W_{-A}^{(1)}\left(\mu_{l}-1 / 2\right)\left(2 \mu_{l}-1\right)}{W_{+1}^{(1)}\left(\mu_{l}-1 / 2\right) W_{-1}^{(1)}\left(\mu_{l}-1 / 2\right)\left(2 \mu_{l}+1\right)} \\
& =\prod_{i=1}^{N} \frac{\left(\mu_{l}-v_{i}-1 / 2\right)\left(\mu_{l}+v_{i}-1 / 2\right)}{\left(\mu_{l}-v_{i}+1 / 2\right)\left(\mu_{l}+v_{i}+1 / 2\right)} \\
& \times\left.\prod_{\substack{i=1 \\
i \neq l}}^{M} \frac{\left(\mu_{l}-\mu_{i}+1\right)\left(\mu_{l}+\mu_{i}+1\right)}{\left(\mu_{l}-\mu_{i}-1\right)\left(\mu_{l}+\mu_{i}-1\right)} \Lambda^{(2)}\left(\tilde{v},\left\{v_{i}\right\},\left\{\mu_{l}\right\}\right)\right|_{\tilde{v}=\mu_{l}} .
\end{aligned}
$$

Here $a=1,2$, and

$$
\begin{gathered}
W_{-A}^{(1)}(v)= \begin{cases}\frac{v+\xi_{-}+1 / 2}{-v+\xi_{-}+1 / 2}, & \text { for } m=3, \\
1, & \text { for } m=1,2,4, \\
\frac{-v+\xi_{-}-1 / 2}{v+\xi_{-}-1 / 2}, & \text { for } m=5,\end{cases} \\
W_{+A}^{(1)}(v)= \begin{cases}\frac{v+3 / 2}{v+1 / 2}, & \text { for } l=1, \\
\frac{(v+3 / 2)\left(v+\xi_{+}-1 / 2\right)}{(v+1 / 2)\left(v+\xi_{+}+1 / 2\right)}, & \text { for } l=2, \\
\frac{(v+3 / 2)\left(v+\xi_{+}+1 / 2\right)}{(v+1 / 2)\left(-v+\xi_{+}-3 / 2\right)}, & \text { for } l=3, \\
\frac{(v+3 / 2)\left(-v+\xi_{+}+1 / 2\right)}{(+1 / 2)\left(-v+\xi_{+}-3 / 2\right)}, & \text { for } l=4, \\
\frac{(v+3 / 2)\left(-v+\xi_{+}-1 / 2\right)}{(v+1 / 2)\left(v+\xi_{+}+3 / 2\right)}, & \text { for } l=5,\end{cases}
\end{gathered}
$$




$$
\begin{aligned}
& W_{-1}^{(1)}(v)=W_{-2}^{(1)}(v)= \begin{cases}\frac{v}{v+1 / 2}, \frac{v}{\frac{v}{(v+1 / 2)\left(v+\xi_{-}-1 / 2\right)},} & \text { for } m=2, \\
\frac{v}{(v+1 / 2)\left(-v+\xi_{-}+1 / 2\right)}, & \text { for } m=3, \\
\frac{v\left(v+\xi_{-}+3 / 2\right)}{(v+1 / 2)\left(-v+\xi_{-}+1 / 2\right)}, & \text { for } m=4, \\
\frac{v}{(v+1 / 2)\left(v+\xi_{-}-1 / 2\right)}, & \text { for } m=5,\end{cases} \\
& W_{+1}^{(1)}(v)=W_{+2}^{(1)}(v)= \begin{cases}\frac{1,}{\frac{1}{v+\xi_{+}+1 / 2},} & \text { for } l=2, \\
\frac{1}{-v+\xi_{+}-3 / 2}, & \text { for } l=3, \\
\frac{v+\xi_{+}+3 / 2}{-v+\xi_{+}-3 / 2}, & \text { for } l=4, \\
\frac{1}{v+\xi_{+}+3 / 2}, & \text { for } l=5 .\end{cases}
\end{aligned}
$$

Now $\Lambda^{(2)}\left(\tilde{v},\left\{v_{i}\right\},\left\{\mu_{l}\right\}\right)$ is the eigenvalue of the third level transfer matrix $\tau^{(2)}$ related to an $S U(2)$ invariant open chain, i.e.

$$
\tau^{(2)}=\operatorname{Tr}_{0} K_{+}^{(2)}(\tilde{v}) T^{(2)}(\tilde{v}) K_{-}^{(2)}(\tilde{v}) \bar{T}^{(2)}(\tilde{v})
$$

where

$$
\begin{aligned}
& T^{(2)}\left(\tilde{v},\left\{v_{i}\right\},\left\{\mu_{l}\right\}\right)=r_{12}^{(2)}\left(\tilde{v}+\mu_{1}\right)_{h_{1} g_{1}}^{e_{1} a} \ldots r_{12}^{(2)}\left(\tilde{v}+\mu_{M}\right)_{h_{M} g_{M}}^{e_{M} g_{M-1}} \\
& \bar{T}^{(1)}\left(\tilde{v},\left\{v_{i}\right\},\left\{\mu_{l}\right\}\right)=r_{21}^{(2)}\left(\tilde{v}-\mu_{M}\right)_{l_{M-1} e_{M} h_{M}}^{e_{M} h_{M}} \ldots r_{21}^{(2)}\left(\tilde{v}-\mu_{1}\right)_{a i_{1}}^{e_{1} h_{1}}
\end{aligned}
$$

\subsection{Third-level Bethe ansatz}

It has been shown so far that the eigenvalue problem of the transfer matrix is reduced to the diagonalization of the isotropic Heisenberg model with $K$ matrices

$$
K_{-}^{(2)}(\tilde{v})= \begin{cases}\left(\begin{array}{cc}
\tilde{v}+\xi_{-}-1 & 0 \\
0 & -\tilde{v}+\xi_{-}-1
\end{array}\right), & \text { for } m=2 \\
\left(\begin{array}{cc}
\tilde{v}+\xi_{-} & 0 \\
0 & -\tilde{v}+\xi_{-}
\end{array}\right), & \text {for } m=3,5 \\
\left(\begin{array}{cc}
1 & 0 \\
0 & 1
\end{array}\right), & \text { for } m=1,4\end{cases}
$$




$$
K_{+}^{(2)}(\tilde{v})= \begin{cases}\left(\begin{array}{cc}
\tilde{v}+\xi_{+} & 0 \\
0 & -\tilde{v}+\xi_{+}
\end{array}\right), & \text {for } l=2, \\
\left(\begin{array}{cc}
\tilde{v}+\xi_{+}+1 & 0 \\
0 & -\tilde{v}+\xi_{+}-1
\end{array}\right), & \text { for } l=3,5 \\
\left(\begin{array}{ll}
1 & 0 \\
0 & 1
\end{array}\right), & \text { for } l=1,4 .\end{cases}
$$

Following the derivation in [17], we immediately obtain the eigenvalues of the nested transfer matrix (80), given by

$$
\begin{aligned}
& \tau^{(2)}(\tilde{v}) F^{(1)^{l_{1} \cdots l_{M}}}=\Lambda^{(2)}\left(v,\left\{v_{i}\right\},\left\{\mu_{l}\right\},\left\{w_{q}\right\}\right) F^{(1)^{l_{1} \cdots l_{M}}} \\
& =\left\{W_{+1}^{(2)}(\tilde{v}) W_{-1}^{(2)}(\tilde{v}) \prod_{l=1}^{Q} \frac{\left(\tilde{v}-w_{l}-1\right)\left(\tilde{v}+w_{l}\right)}{\left(\tilde{v}-w_{l}\right)\left(\tilde{v}+w_{l}+1\right)}\right. \\
& +W_{+2}^{(2)}(\tilde{v}) W_{-2}^{(2)}(\tilde{v}) \prod_{l=1}^{M} \frac{\left(\tilde{v}-\mu_{l}\right)\left(\tilde{v}+\mu_{l}\right)}{\left(\tilde{v}-\mu_{l}+1\right)\left(\tilde{v}+\mu_{l}+1\right)} \\
& \left.\times \prod_{l=1}^{Q} \frac{\left(\tilde{v}-w_{l}+1\right)\left(\tilde{v}+w_{l}+2\right)}{\left(\tilde{v}-w_{l}\right)\left(\tilde{v}+w_{l}+1\right)}\right\} F^{(1)^{l_{1} \cdots l_{M}}}
\end{aligned}
$$

provided that

$$
\begin{aligned}
& \frac{W_{+1}^{(2)}\left(w_{l}\right) W_{-1}^{(2)}\left(w_{l}\right) w_{l}}{W_{+2}^{(2)}\left(w_{l}\right) W_{-2}^{(2)}\left(w_{l}\right)\left(w_{l}+1\right)}=\prod_{j=1}^{M} \frac{\left(w_{l}-\mu_{j}\right)\left(w_{l}+\mu_{j}\right)}{\left(w_{l}-\mu_{j}+1\right)\left(w_{l}+\mu_{j}+1\right)} \\
& \times \prod_{\substack{m=1 \\
m \neq l}}^{Q} \frac{\left(w_{l}-w_{m}+1\right)\left(w_{l}+w_{m}+2\right)}{\left(w_{l}-w_{m}-1\right)\left(w_{l}+w_{m}\right)} .
\end{aligned}
$$

Here

$$
\begin{aligned}
& W_{-1}^{(2)}(\tilde{v})= \begin{cases}\tilde{v}+\xi_{-}-1, & \text { for } m=2, \\
\tilde{v}+\xi_{-}, & \text {for } m=3,5, \\
1, & \text { for } m=1,4,\end{cases} \\
& W_{+1}^{(2)}(\tilde{v})= \begin{cases}\frac{(\tilde{v}+1)\left(\tilde{v}+\xi_{+}-1\right)}{2 \tilde{v}+1}, & \text { for } l=2, \\
\frac{(\tilde{v}+1)\left(\tilde{v}+\xi_{+}\right)}{2 \tilde{v}+1}, & \text { for } l=3,5, \\
\frac{\tilde{v}+1}{\tilde{v}+1 / 2}, & \text { for } l=1,4,\end{cases} \\
& W_{-2}^{(2)}(\tilde{v})= \begin{cases}\frac{-\tilde{v}\left(\tilde{v}-\xi_{-}+2\right)}{\tilde{v}+1 / 2}, & \text { for } m=2, \\
\frac{-\tilde{v}\left(\tilde{v}-\xi_{-}+1\right)}{\tilde{v}+1 / 2}, & \text { for } m=3,5, \\
\frac{\tilde{v}}{\tilde{v}+1 / 2}, & \text { for } m=1,4,\end{cases}
\end{aligned}
$$




$$
W_{+2}^{(2)}(\tilde{v})=\left\{\begin{array}{l}
-\tilde{v}+\xi_{+}-2, \text { for } \quad l=2, \\
-\tilde{v}+\xi_{+}-1, \text { for } l=3,5, \\
1, \quad \text { for } l=1,4 .
\end{array}\right.
$$

The eigenvalues (74) and (85) as well as the constraints (75) (86) on the rapidities $\mu_{l}$ and $w_{l}$ have paved the way for the complete diagonalization of the transfer matrix (25). Making a further shift on the rapidities, $w_{l} \rightarrow w_{l}-1 / 2$, $w_{m} \rightarrow w_{m}-1 / 2$ and $\tilde{v}=v+1 / 2$, the eigenvalues of the transfer matrix (25) are given by

$$
\begin{aligned}
& \Lambda\left(v,\left\{v_{i}\right\},\left\{\mu_{l}\right\},\left\{w_{j}\right\}\right)=K 1_{-}\left(v-\frac{1}{2}\right) K 1_{+}\left(v-\frac{1}{2}\right) \\
& \left\{W_{A}^{+}\left(v-\frac{1}{2}\right) W_{A}^{-}\left(v-\frac{1}{2}\right)\left(v+\frac{1}{2}\right)^{2 L}\right. \\
& \times \prod_{i=1}^{N} \frac{\left(v-v_{i}-1\right)\left(v+v_{i}-1\right)}{\left(v-v_{i}\right)\left(v+v_{i}\right)} \\
& +W_{1}^{+}\left(v-\frac{1}{2}\right) W_{1}^{-}\left(v-\frac{1}{2}\right) W_{+A}^{(1)}(v) W_{-A}^{(1)}(v)\left(v-\frac{1}{2}\right)^{2 L} \\
& \times \prod_{i=1}^{N} \frac{\left(v-v_{i}+1\right)\left(v+v_{i}+1\right)}{\left(v-v_{i}\right)\left(v+v_{i}\right)} \prod_{l=1}^{M} \frac{\left(v-u_{l}-\frac{1}{2}\right)\left(v+u_{l}-\frac{1}{2}\right)}{\left(v-u_{l}+\frac{1}{2}\right)\left(v+u_{l}+\frac{1}{2}\right)} \\
& +W_{2}^{+}\left(v-\frac{1}{2}\right) W_{2}^{-}\left(v-\frac{1}{2}\right) W_{+1}^{(1)}(v) W_{-1}^{(1)}(v) W_{+1}^{(2)}\left(v+\frac{1}{2}\right) W_{-1}^{(2)}\left(v+\frac{1}{2}\right)\left(v-\frac{1}{2}\right)^{2 L} \\
& \times \prod_{l=1}^{M} \frac{\left(v-\mu_{l}+\frac{3}{2}\right)\left(v+\mu_{l}+\frac{3}{2}\right)}{\left(v-\mu_{l}+\frac{1}{2}\right)\left(v+\mu_{l}+\frac{1}{2}\right)} \prod_{j=1}^{Q} \frac{\left(v-w_{j}\right)\left(v+w_{j}\right)}{\left(v-w_{j}+1\right)\left(v+w_{l}+1\right)} \\
& +W_{3}^{+}\left(v-\frac{1}{2}\right) W_{3}^{-}\left(v-\frac{1}{2}\right) W_{+2}^{(1)}(v) W_{-2}^{(1)}(v) W_{+2}^{(2)}\left(v+\frac{1}{2}\right) W_{-2}^{(2)}\left(v+\frac{1}{2}\right)\left(v-\frac{1}{2}\right)^{2 L} \\
& \left.\times \prod_{j=1}^{Q} \frac{\left(v-w_{j}+2\right)\left(v+w_{j}+2\right)}{\left(v-w_{j}+1\right)\left(v+w_{l}+1\right)}\right\} .
\end{aligned}
$$

The three rapidities $\left\{v_{i}, \mu_{j}, w_{k}\right\}$ of flavor waves satisfy the Bethe ansatz equations

$$
\begin{aligned}
& \zeta\left(v_{i}, \xi_{+}\right) \zeta\left(v_{i}, \xi_{-}\right) \frac{\left(v_{i}+\frac{1}{2}\right)^{2 L}}{\left(v_{i}-\frac{1}{2}\right)^{2 L}} \\
& =\prod_{\substack{l=1 \\
l \neq i}}^{N} \frac{\left(v_{i}-v_{l}+1\right)\left(v_{i}+v_{l}+1\right)}{\left(v_{i}-v_{l}-1\right)\left(v_{i}+v_{l}-1\right)} \prod_{l=1}^{M} \frac{\left(v_{i}-\mu_{l}-\frac{1}{2}\right)\left(v_{i}+\mu_{l}-\frac{1}{2}\right)}{\left(v_{i}-\mu_{l}+\frac{1}{2}\right)\left(v_{i}+\mu_{l}+\frac{1}{2}\right)} \\
& \eta\left(\mu_{j}, \xi_{+}\right) \eta\left(\mu_{j}, \xi_{-}\right) \prod_{i=1}^{N} \frac{\left(\mu_{j}-v_{i}+\frac{1}{2}\right)\left(\mu_{j}+v_{i}+\frac{1}{2}\right)}{\left(\mu_{j}-v_{i}-\frac{1}{2}\right)\left(\mu_{j}+v_{i}-\frac{1}{2}\right)}
\end{aligned}
$$




$$
\begin{aligned}
& =\prod_{\substack{i=1 \\
i \neq j}}^{M} \frac{\left(\mu_{j}-\mu_{i}+1\right)\left(\mu_{j}+\mu_{i}+1\right)}{\left(\mu_{j}-\mu_{i}-1\right)\left(\mu_{j}+\mu_{i}-1\right)} \prod_{l=1}^{Q} \frac{\left(\mu_{j}-w_{l}-\frac{1}{2}\right)\left(\mu_{j}+w_{l}-\frac{1}{2}\right)}{\left(\mu_{j}-w_{l}+\frac{1}{2}\right)\left(\mu_{j}+w_{l}+\frac{1}{2}\right)} \\
& \Omega\left(w_{k}, \xi_{+}\right) \Omega\left(w_{k}, \xi_{-}\right) \prod_{l=1}^{M} \frac{\left(w_{k}-\mu_{l}+\frac{1}{2}\right)\left(w_{k}+\mu_{l}+\frac{1}{2}\right)}{\left(w_{k}-\mu_{l}-\frac{1}{2}\right)\left(w_{k}+\mu_{l}-\frac{1}{2}\right)} \\
& =\prod_{\substack{l=1 \\
l \neq k}}^{Q} \frac{\left(w_{k}-w_{l}+1\right)\left(w_{k}+w_{l}+1\right)}{\left(w_{k}-w_{l}-1\right)\left(w_{k}+w_{l}-1\right)}
\end{aligned}
$$

for $i=1, \ldots, N, j=1, \ldots, M$ and $k=1, \ldots, Q$, respectively. Here, we have introduced the notation

$$
\begin{aligned}
& \zeta\left(v_{i}, \xi_{ \pm}\right)= \begin{cases}\frac{v_{i}+\xi_{ \pm}-\frac{1}{2}}{v_{i}-\xi_{ \pm}+\frac{1}{2}}, \text { for } l=1, \quad & m=1, \\
\frac{v_{i}-\xi_{ \pm}-\frac{1}{2}}{v_{i}+\xi_{ \pm}+\frac{1}{2}}, & \text { for } l=3, \quad m=3, \\
1, & \text { for } l=2,4, \quad m=2,4, \\
\frac{v_{i}+\xi_{ \pm}-\frac{1}{2}}{v_{i}-\xi_{ \pm}+\frac{1}{2}}, & \text { for } l=5, \quad m=5,\end{cases} \\
& \eta\left(\mu_{j}, \xi_{ \pm}\right)=\left\{\begin{array}{lll}
1, & \text { for } l=1,2,3, \quad m=1,2,3 \\
\frac{\mu_{j}+\xi_{ \pm}-\frac{1}{2}}{\mu_{j}-\xi_{ \pm}+\frac{1}{2}}, & \text { for } l=4, \quad m=4, \\
\frac{\mu_{j}-\xi_{ \pm}}{\mu_{j}+\xi_{ \pm}}, & \text {for } l=5, \quad m=5,
\end{array}\right. \\
& \Omega\left(w_{k}, \xi_{ \pm}\right)= \begin{cases}\frac{w_{k}+\xi_{ \pm}-\frac{3}{2}}{w_{k}-\xi_{ \pm}+\frac{3}{2}}, \text { for } l=2, \quad m=2, \\
\frac{w_{k}+\xi_{ \pm}-\frac{1}{2}}{w_{k}-\xi_{ \pm}+\frac{1}{2}}, \text { for } l=3,5, \quad m=3,5, \\
1, & \text { for } l=1,4, \quad m=1,4 .\end{cases}
\end{aligned}
$$

These boundary factors coupled to the three degrees of freedom will result in a rich physical scenario. From (27) and (91), we finally obtain the eigenvalues of the Hamiltonian (1) as

$$
E=\left\{\begin{array}{l}
\frac{U_{+}}{2}+\frac{U_{-}}{2}+\left(\frac{J_{\|}}{\gamma}-J_{\perp}\right) L \\
+\sum_{i=1}^{N}\left(\frac{J_{\|}}{\gamma} \frac{1}{v_{i}^{2}-\frac{1}{4}}+J_{\perp}\right), \text { for } l=m=1,2,5, \\
-\frac{U_{+}}{2}-\frac{U_{-}}{2}+\left(\frac{J_{\|}}{\gamma}-J_{\perp}\right) L \\
+\sum_{i=1}^{N}\left(\frac{J_{\|}}{\gamma} \frac{1}{v_{i}^{2}-\frac{1}{4}}+J_{\perp}\right), \text { for } l=m=3,4 .
\end{array}\right.
$$




\section{Boundary impurity effects}

Having diagonalised the Hamiltonian (1) by means of the algebraic Bethe ansatz, the next step is to derive the thermodynamic Bethe ansatz equations.

\subsection{Derivation of TBA}

For later convenience in the analysis of the Bethe ansatz equations, we make the change of variables: $v_{i} \rightarrow-\mathrm{i} v_{i}, \mu_{l} \rightarrow-\mathrm{i} \mu_{l}, w_{k} \rightarrow-\mathrm{i} w_{k}$ and some rescalings in the boundary parameters $\xi_{ \pm}$. The Bethe ansatz equations are now

$$
\begin{aligned}
& \zeta\left(v_{i}, \beta_{ \pm}\right) \prod_{r= \pm} \prod_{\substack{l=1 \\
l \neq i}}^{N} \frac{v_{i}-r v_{l}-\mathrm{i}}{v_{i}-r v_{l}+\mathrm{i}} \prod_{l=1}^{M} \frac{v_{i}-r \mu_{l}+\frac{\mathrm{i}}{2}}{v_{i}-r \mu_{l}-\frac{\mathrm{i}}{2}}=\frac{\left(v_{i}-\frac{\mathrm{i}}{2}\right)^{2 L}}{\left(v_{i}+\frac{\mathrm{i}}{2}\right)^{2 L}} \\
& \eta\left(\mu_{j}, \beta_{ \pm}\right) \prod_{r= \pm} \prod_{\substack{i=1 \\
i \neq j}}^{M} \frac{\mu_{j}-r \mu_{i}-\mathrm{i}}{\mu_{j}-r \mu_{i}+\mathrm{i}} \prod_{l=1}^{Q} \frac{\mu_{j}-r w_{l}+\frac{\mathrm{i}}{2}}{\mu_{j}-r w_{l}-\frac{\mathrm{i}}{2}} \prod_{i=1}^{N} \frac{\mu_{j}-r v_{i}+\frac{\mathrm{i}}{2}}{\mu_{j}-r v_{i}-\frac{\mathrm{i}}{2}}=1, \\
& \Omega\left(w_{k}, \beta_{ \pm}\right) \prod_{r= \pm} \prod_{\substack{l=1 \\
l \neq k}}^{Q} \frac{w_{k}-r w_{l}-\mathrm{i}}{w_{k}-r w_{l}+\mathrm{i}} \prod_{l=1}^{M} \frac{w_{k}-r \mu_{l}+\frac{\mathrm{i}}{2}}{w_{k}-r \mu_{l}-\frac{\mathrm{i}}{2}}=1
\end{aligned}
$$

where

$$
\begin{aligned}
& \zeta\left(v_{i}, \beta_{ \pm}\right)= \begin{cases}\frac{v_{i}+\mathrm{i} \beta_{ \pm}}{v_{i}-\mathrm{i} \beta_{ \pm}}, & \text {for } l=1,3,5, \quad m=1,3,5 \\
1, & \text { for } l=2,4, \quad m=2,4\end{cases} \\
& \eta\left(\mu_{j}, \beta_{ \pm}\right)= \begin{cases}1, & \text { for } l=1,2,3, \quad m=1,2,3, \\
\frac{\mu_{j}+\mathrm{i} \beta_{ \pm}}{\mu_{j}-\mathrm{i} \beta_{ \pm}}, & \text {for } l=4, \quad m=4, \\
\frac{\mu_{j}-\mathrm{i}\left(\beta_{ \pm}+\frac{1}{2}\right)}{\mu_{j}+\mathrm{i}\left(\beta_{ \pm}+\frac{1}{2}\right)}, & \text { for } l=5, \quad m=5,\end{cases} \\
& \Omega\left(w_{k}, \beta_{ \pm}\right)= \begin{cases}\frac{w_{k}+\mathrm{i} \beta_{ \pm}}{w_{k}-\mathrm{i} \beta_{ \pm}}, & \text {for } l=2,5, \quad m=2,5 \\
\frac{w_{k}-\mathrm{i}\left(\beta_{ \pm}+1\right)}{w_{k}+\mathrm{i}\left(\beta_{ \pm}+1\right)}, & \text { for } l=3, \quad m=3 \\
1, & \text { for } l=1,4, \quad m=1,4 .\end{cases}
\end{aligned}
$$

The shifts in the parameters $\xi_{ \pm}$are given by 


$$
\begin{array}{ll}
\beta_{ \pm}=\xi_{ \pm}-\frac{1}{2}, & \text { for } l=m=1,4,5, \\
\beta_{ \pm}=\xi_{ \pm}-\frac{3}{2}, & \text { for } l=m=2, \\
\beta_{ \pm}=-\xi_{ \pm}-\frac{1}{2}, & \text { for } l=m=3 .
\end{array}
$$

Correspondingly, the energy spectrum is given by

$$
E=\sum_{i=1}^{N}\left(-\frac{J_{\|}}{\gamma} \frac{1}{v_{i}^{2}+\frac{1}{4}}+J_{\perp}\right) .
$$

Here we have dropped some constants appearing in Eq. (98), which will be used in deriving one point correlation functions later.

From the above Bethe ansatz equations (99)-(101), it is found that in the cases $l=m=1,3,5$, the solutions $v_{1}= \pm \mathrm{i} \beta_{-}$and $v_{\mathrm{r}}= \pm \mathrm{i} \beta_{+}$form two boundary bound sates in the charge rapidity when $\beta_{ \pm}$are negative. Nevertheless, in the case $l=m=5$, besides the charge boundary bound states, the boundary bound states exist also in the spin rapidites, i.e.

$$
\mu=\left\{\begin{array}{l} 
\pm \mathrm{i}\left(\beta_{-}+\frac{1}{2}\right), \\
\pm \mathrm{i}\left(\beta_{+}+\frac{1}{2}\right),
\end{array} \quad w=\left\{\begin{array}{l} 
\pm \mathrm{i} \beta_{-} \\
\pm \mathrm{i} \beta_{+}
\end{array}\right.\right.
$$

No boundary bound state exists in the remaining cases. We observe that when $J_{\perp}>\frac{2 J_{\|}}{\gamma}(1-\cos k)$, the reference state becomes the true ground state, i.e., the ground state is given by a product of the singlet rung states. The minimal gap can be easily calculated and is given by

$$
\Delta=J_{\perp}-\frac{2 J_{\|}}{\gamma}(1-\cos k)
$$

where $k=\pi /\left[1+\frac{1}{4 L}\left(\frac{1}{\beta_{+}}+\frac{1}{\beta_{-}}\right)\right]$. It is obvious that gap remains almost unchanged in the thermodynamic limit and is almost the same as $\Delta=J_{\perp}-\frac{4 J_{\|}}{\gamma}$ in the periodic case because $L>>\frac{1}{\beta_{ \pm}}$. In the regime $-\frac{1}{2}<\beta_{ \pm}<-\frac{1}{2} \sqrt{1-\frac{4 J_{\|}}{\gamma J_{\perp}}}$, the boundary bound states are stable. Otherwise, in the remaining regime, they become excited states. In the limit $J_{\perp} \rightarrow \infty$, all the boundary bound states are excitations. We shall see that the boundary bound states radically affect the edge ground state properties. For $J_{c}^{-}=-\frac{J_{\|}}{\gamma}\left(\frac{\pi}{\sqrt{3}}-\ln 3\right)<J_{\perp}<\frac{4 J_{\|}}{\gamma}$, the ground state consists of three branches of Luttinger liquids associated with the rapidities $v, \mu$ and $w$. Here $J_{c}^{-}$is the critical transition point from the $S U(3)$ phase into the $S U(4)$ phase in the absence of a magnetic field. The triplet states can exist in the ground state. This corresponds to a continuum of massless excitations. 
The thermodynamics of the boundary fields can be derived from the Bethe ansatz equations (99)-(101). We now focus on the analysis of the Bethe ansatz equations. As usual, we define the functions

$$
\begin{aligned}
& e_{n}(x)=\frac{x+\mathrm{i} \frac{n}{2}}{x-\mathrm{i} \frac{n}{2}}, \quad \theta_{n}(x)=\mathrm{i} \ln e_{n}(x), \\
& a_{n}(x)=\frac{1}{2 \pi} \frac{n}{x^{2}+\frac{n^{2}}{4}} \equiv \frac{1}{2 \pi} \frac{d}{d x} \theta_{n}(x),
\end{aligned}
$$

in terms of which the Bethe ansatz equations (99)-(101) become

$$
\begin{aligned}
& \zeta\left(v_{i}, \beta_{ \pm}\right)\left(e_{1}\left(v_{i}\right)\right)^{2 L} \prod_{r= \pm} \prod_{l=1}^{M} e_{1}\left(v_{i}-r \mu_{l}\right)=\prod_{r= \pm} \prod_{\substack{l=1 \\
l \neq i}}^{N} e_{2}\left(v_{i}-r v_{l}\right) \\
& \eta\left(\mu_{j}, \beta_{ \pm}\right) \prod_{r= \pm} \prod_{l=1}^{N} e_{1}\left(\mu_{j}-r v_{l}\right)=\prod_{\substack { r= \pm{c}{l=1 \\
l \neq j{ r = \pm \\
\begin{subarray} { c } { l = 1 \\
l \neq j } }\end{subarray}}^{M} e_{2}\left(\mu_{j}-r \mu_{l}\right) \prod_{l=1}^{Q} e_{-1}\left(\mu_{j}-r w_{l}\right), \\
& \Omega\left(w_{k}, \beta_{ \pm}\right) \prod_{r= \pm} \prod_{l=1}^{M} e_{1}\left(w_{k}-r \mu_{l}\right)=\prod_{r= \pm} \prod_{\substack{l=1 \\
l \neq k}}^{Q} e_{2}\left(w_{k}-r w_{l}\right)
\end{aligned}
$$

In order to study the thermodynamics of the model with boundary impurities we begin by adopting the string hypothesis [26]. If we define $v_{-j}=-v_{j}$, $\mu_{-l}=-\mu_{l}$ and $w_{-k}=w_{k}$, the Bethe ansatz equations (99)-(101) admit the string solutions

$$
\begin{aligned}
v_{\alpha_{1} j}^{n} & =v_{\alpha_{1}}^{n}+\mathrm{i} \frac{1}{2}(n+1-2 j), \\
\mu_{\alpha_{2} j}^{n} & =\mu_{\alpha_{2}}^{n}+\mathrm{i} \frac{1}{2}(n+1-2 j), \\
w_{\alpha_{3} j}^{n} & =w_{\alpha_{3}}^{n}+\mathrm{i} \frac{1}{2}(n+1-2 j),
\end{aligned}
$$

in thermodynamic limit, where $j=1, \cdots, n, \alpha_{a}=1, \cdots, N_{n}^{(a)}$ and $v_{\alpha_{1}}^{n}, \mu_{\alpha_{2}}^{n}$ and $w_{\alpha_{3}}^{n}$ are the positions of the center of the strings. The number of $n$-strings $N_{n}^{(a)}$ satisfy the relation $P^{(a)}=\sum_{n} n N_{n}^{(a)}$. By taking the thermodynamic limit, the Bethe ansatz equations become

$$
\begin{aligned}
& \rho_{n}^{(1) h}=a_{n}+\frac{1}{2 L} \rho_{\mathrm{b} n}^{(1)}-\sum_{m} A_{n m} * \rho_{m}^{(1)}+\sum_{m} a_{n m} * \rho_{m}^{(2)}, \\
& \rho_{n}^{(2) h}=\frac{1}{2 L} \rho_{\mathrm{b} n}^{(2)}-\sum_{m} A_{n m} * \rho_{m}^{(2)}+\sum_{m} a_{n m} *\left(\rho_{m}^{(1)}+\rho_{m}^{(3)}\right),
\end{aligned}
$$




$$
\rho_{n}^{(3) h}=\frac{1}{2 L} \rho_{\mathrm{bn}}^{(3)}-\sum_{m} A_{n m} * \rho_{m}^{(3)}+\sum_{m} a_{n m} * \rho_{m}^{(2)}
$$

where the symbol $*$ denotes the usual convolution. Here $\rho_{n}^{(a)}(v), a=1,2,3$ are the densities of roots of the three flavors, $\rho_{n}^{(a) h}(v), a=1,2,3$ are the densities of holes of the three flavors and $\rho_{\mathrm{b} n}^{(i)}, i=1,2,3$ are the contributions from boundary fields associated with different rapidities. These boundary phase factors are given by

$$
\begin{aligned}
& \rho_{\mathrm{b} n}^{(1)}= \begin{cases}\sum_{ \pm} \sum_{l=1}^{n} a_{n+2 \beta_{ \pm}+1-2 l}(\lambda)+a_{n 2}(\lambda), & \text { for } l=1,3,5, \quad m=1,3,5, \\
a_{n 2}(\lambda), & \text { for } l=2,4, \quad m=2,4,\end{cases} \\
& \rho_{\mathrm{b}}^{(2)}= \begin{cases}a_{n 2}(\lambda)-a_{n 1}(\lambda), & \text { for } l=1,2,3, m=1,2,3, \\
\sum_{ \pm} \sum_{l=1}^{n} a_{n+2 \beta_{ \pm}+1-2 l}(\lambda)+a_{n 2}(\lambda)-a_{n 1}(\lambda), & \text { for } l=4, m=4, \\
-\sum_{116}\end{cases} \\
& \rho_{\mathrm{b} n}^{(2)}= \begin{cases}\sum_{ \pm} \sum_{l=1}^{n} a_{n+2 \beta_{ \pm}+1-2 l}(\lambda)+a_{n 2}(\lambda)-a_{n 1}(\lambda), & \text { for } l=4, \\
-\sum_{ \pm} \sum_{l=1}^{n} a_{n+2 \beta_{ \pm}+2-2 l}(\lambda) & \text { for } l=5, \quad m=5, \\
+a_{n 2}(\lambda)-a_{n 1}(\lambda), & m,\end{cases} \\
& \rho_{\mathrm{b} n}^{(3)}= \begin{cases}\sum_{ \pm} \sum_{l=1}^{n} a_{n+2 \beta_{ \pm}+1-2 l}(\lambda)+a_{n 2}(\lambda), & \text { for } l=2,5, \quad m=2,5, \\
-\sum_{ \pm} \sum_{l=1}^{n} a_{n+2 \beta_{ \pm}+3-2 l}(\lambda)+a_{n 2}(\lambda), & \text { for } l=3, \quad m=3, \\
a_{n 2}(\lambda), & \text { for } l=1,4, \quad m=1,4 .\end{cases}
\end{aligned}
$$

In addition

$$
\begin{aligned}
& A_{n m}(\lambda)=\delta(\lambda) \delta_{n m}+\left(1-\delta_{n m}\right) a_{|n-m|}(\lambda)+a_{n+m}(\lambda) \\
&+2 \sum_{l=1}^{\operatorname{Min}(n, m)-1} a_{|n-m|+2 l}(\lambda) \\
& a_{n m}(\lambda)=\sum_{l=1}^{\operatorname{Min}(n, m)} a_{n+m+1-2 l}(\lambda) .
\end{aligned}
$$

We emphasize that the boundary potentials enter in the expression for the ground state energy implicitly via $\rho_{\mathrm{b}}^{(a)}(v)$ in the above equations, with contributions to the densities of the roots at the order of $1 / L$. In order to find the equilibrium state of the system at fixed temperature $T$ and external magnetic field $H(\geq 0)$, we minimize the free energy $F=E-T S-H S^{z}$ with respect to the densities to obtain the TBA in the form

$$
\left(\begin{array}{c}
\ln \left(1+\eta_{n}^{(1)}\right) \\
\ln \left(1+\eta_{n}^{(2)}\right) \\
\ln \left(1+\eta_{n}^{(3)}\right)
\end{array}\right)=\frac{G_{n}}{T}+\left(\begin{array}{ccc}
\sum_{m} A_{n m} & -\sum_{m} a_{n m} & 0 \\
-\sum_{m} a_{n m} & \sum_{m} A_{n m} & -\sum_{m} a_{n m} \\
0 & -\sum_{m} a_{n m} & \sum_{m} A_{n m}
\end{array}\right) *\left(\begin{array}{c}
\ln \left(1+\frac{1}{\eta_{m}^{(1)}}\right) \\
\ln \left(1+\frac{1}{\eta_{m}^{(2)}}\right) \\
\ln \left(1+\frac{1}{\eta_{m}^{(3)}}\right)
\end{array}\right)
$$


The driving matrix $G_{n}$ depends on the choice of the reference state. Explicitly, for $J_{\perp}<0, G=\operatorname{column}\left(-\frac{J_{\|}}{\gamma} 2 \pi a_{n}+n H, n H,-n\left(J_{\perp}+H\right)\right)$, giving the free energy

$$
\begin{aligned}
\frac{F(T, H)}{2 L}= & -H-T \int_{-\infty}^{\infty} \sum_{n=1}^{\infty} a_{n}(\lambda) \ln \left(1+e^{-\frac{\epsilon_{n}^{(1)}(\lambda)}{T}}\right) d \lambda \\
& -\frac{T}{2 L} \sum_{a=1}^{3} \int_{-\infty}^{\infty} \sum_{n=1}^{\infty} \rho_{\mathrm{b} n}^{(a)} \ln \left(1+e^{-\frac{\epsilon_{n}^{(a)}(\lambda)}{T}}\right) d \lambda .
\end{aligned}
$$

On the other hand, for $J_{\perp}>0, G=\operatorname{colum}\left(-\frac{J_{\|}}{\gamma} 2 \pi a_{n}+n\left(J_{\perp}-H\right), n H, n H\right)$ and the free energy is given by

$$
\begin{aligned}
\frac{F(T, H)}{2 L}= & -T \int_{-\infty}^{\infty} \sum_{n=1}^{\infty} a_{n}(\lambda) \ln \left(1+e^{-\frac{\epsilon_{n}^{(1)}(\lambda)}{T}}\right) d \lambda \\
& -\frac{T}{2 L} \sum_{a=1}^{3} \int_{-\infty}^{\infty} \sum_{n=1}^{\infty} \rho_{\mathrm{bn}}^{(a)} \ln \left(1+e^{-\frac{\epsilon_{n}^{(a)}(\lambda)}{T}}\right) d \lambda .
\end{aligned}
$$

Here $\eta_{n}^{(l)}(\lambda)=\rho^{(l) h}(\lambda) / \rho^{(l)}(\lambda) \equiv \exp \left(\epsilon_{n}^{(l)}(\lambda) / T\right), l=1,2,3$, with the dressed energy $\epsilon_{n}^{(l)}$ playing the role of an excitation energy measured from the Fermi level.

Using the relations,

$$
\begin{aligned}
\left(a_{0}+a_{2}\right) * \ln \eta_{n}^{(a)}= & a_{1} *\left[\ln \left(1+\eta_{n+1}^{(a)}\right)+\ln \left(1+\eta_{n-1}^{(a)}\right)\right] \\
& -\ln \left(1+\frac{1}{\eta_{n}^{(a-1)}}\right)-\ln \left(1+\frac{1}{\eta_{n}^{(a+1)}}\right)
\end{aligned}
$$

another form of the TBA is given by

$$
\begin{aligned}
\epsilon_{1}^{(a)}= & g_{1}^{(a)}+T a_{2} * \ln \left(1+e^{-\frac{\epsilon_{1}^{(a)}}{T}}\right)+T\left(a_{0}+a_{2}\right) \sum_{m=1}^{\infty} a_{m} * \ln \left(1+e^{-\frac{\epsilon_{m+1}^{(a)}}{T}}\right) \\
& -T \sum_{m=1}^{\infty} a_{m} *\left(\ln \left(1+e^{-\frac{\epsilon_{m}^{(a-1)}}{T}}\right)+\ln \left(1+e^{-\frac{\epsilon_{m}^{(a+1)}}{T}}\right)\right) \\
\epsilon_{n}^{(a)}= & g_{n}^{(a)}+T a_{1} * \ln \left(1+e^{\frac{\epsilon_{n-1}^{(a)}}{T}}\right) \\
& +T a_{2} * \ln \left(1+e^{-\frac{\epsilon_{n}^{(a)}}{T}}\right)+T\left(a_{0}+a_{2}\right) \sum_{m \geq n}^{\infty} a_{m-n} * \ln \left(1+e^{-\frac{\epsilon_{m}^{(a)}}{T}}\right)
\end{aligned}
$$




$$
-T \sum_{m \geq n}^{\infty} a_{m-n+1} *\left(\ln \left(1+e^{-\frac{\epsilon_{m}^{(a-1)}}{T}}\right)+\ln \left(1+e^{-\frac{\epsilon_{m}^{(a+1)}}{T}}\right)\right),
$$

for $n \geq 2$. In the above $a=1,2,3$ and $\epsilon_{n}^{(0)}(\lambda)=\epsilon_{n}^{(4)}(\lambda)=0$ is assumed. The driving terms are given explicitly by

$$
\begin{aligned}
& g_{1}^{(1)}=-\frac{J_{\|}}{\gamma} 2 \pi a_{1}+H, \quad g_{n}^{(1)}=H, \\
& g_{1}^{(2)}=H, \quad g_{n}^{(2)}=H, \quad \text { for } \quad J_{\perp}<0, \\
& g_{1}^{(3)}=-\left(J_{\perp}+H\right), \quad g_{n}^{(3)}=-\left(J_{\perp}+H\right), \\
& g_{1}^{(1)}=-\frac{J_{\|}}{\gamma} 2 \pi a_{1}+J_{\perp}-H, \quad g_{n}^{(1)}=J_{\perp}-H, \\
& g_{1}^{(2)}=H, \quad g_{n}^{(2)}=H, \quad \text { for } J_{\perp} \geq 0 . \\
& g_{1}^{(3)}=H, \quad g_{n}^{(3)}=H \text {. }
\end{aligned}
$$

\subsection{Boundary bound states and impurity effects}

In the low temperature limit, the states with positive dressed energy are empty. The zeros of the dressed energies define the fermi energies. We decompose $\epsilon_{n}^{(a)}$ into its positive and negative parts, $\epsilon_{n}^{(a)}=\epsilon_{n}^{(a)+}+\epsilon_{n}^{(a)-}$. An analysis of equations (122) and (123) in the limit $T \rightarrow 0$ reveals that for the ground state, the roots are all real corresponding to $n=1$. All dressed energies $\epsilon_{n}^{(a)+}$ with $n \geq 2$ correspond to excitations. Thus the TBA for the ground state is, for $J_{\perp}<0$,

$$
\begin{aligned}
& \epsilon^{(1)}=g_{1}^{(1)}-a_{2} * \epsilon^{(1)-}+a_{1} * \epsilon^{(2)-}, \\
& \epsilon^{(2)}=H-a_{2} * \epsilon^{(2)-}+a_{1} *\left[\epsilon^{(1)-}+\epsilon^{(3)-}\right], \\
& \epsilon^{(3)}=-H-J_{\perp}-a_{2} * \epsilon^{(3)-}+a_{1} * \epsilon^{(2)-},
\end{aligned}
$$

and for $J_{\perp} \geq 0$,

$$
\begin{aligned}
& \epsilon^{(1)}=g_{1}^{(1)}-a_{2} * \epsilon^{(1)-}+a_{1} * \epsilon^{(2)-}, \\
& \epsilon^{(2)}=H-a_{2} * \epsilon^{(2)-}+a_{1} *\left[\epsilon^{(1)-}+\epsilon^{(3)-}\right], \\
& \epsilon^{(3)}=H-a_{2} * \epsilon^{(3)-}+a_{1} * \epsilon^{(2)-} .
\end{aligned}
$$

In this case, the free energy is given by

$$
\frac{F(0, H)}{2 L}= \begin{cases}-H+\int_{-\infty}^{\infty} a_{1}(\lambda) \epsilon_{1}^{(1)-}(\lambda) d \lambda+\frac{1}{2 L} f_{\mathrm{b}}, & \text { for } \quad J_{\perp}<0 \\ \int_{-\infty}^{\infty} a_{1}(\lambda) \epsilon_{1}^{(1)-}(\lambda) d \lambda+\frac{1}{2 L} f_{\mathrm{b}}, & \text { for } J_{\perp} \geq 0\end{cases}
$$


where

$$
f_{\mathrm{b}}=\sum_{a=1}^{3} \int_{-Q_{a}}^{Q_{a}} \rho_{\mathrm{b} 1}^{(a)}(\lambda) \epsilon_{1}^{(a)-}(\lambda)+\theta\left(\beta_{ \pm}+\beta_{c}\right) E_{\mathrm{bs}}
$$

and $\theta(x)$ denotes a step-like function. Define $\beta_{c}=-\frac{1}{2} \sqrt{1-\frac{4 J_{\|}}{\gamma J_{\perp}}}$, then in the interval $-\frac{1}{2}<\beta<\beta_{c}, \theta(\beta)=1$, else $\theta(\beta)=0$. In the above $E_{\mathrm{bs}}$ denotes the boundary bound state energy, given by

$$
E_{\mathrm{bs}}= \begin{cases}\sum_{ \pm}\left(-\frac{J_{\|}}{\gamma} \frac{1}{-\beta_{ \pm}^{2}+\frac{1}{4}}+J_{\perp}\right), & \text { for } J_{\perp} \geq 0 \\ \sum_{ \pm}\left(-\frac{J_{\|}}{\gamma} \frac{1}{-\beta_{ \pm}^{2}+\frac{1}{4}}\right), & \text { for } J_{\perp}<0\end{cases}
$$

It is worth noticing that if $\beta_{ \pm}<\beta_{c}$ we should take the boundary bound states into account in the boundary contributions $\rho_{\mathrm{b} 1}^{(a)}$ for the cases $l=m=1,3,5$. The TBA (126) and (127) provide a clear physical picture of the groundstate and in turn the thermodynamic properties, such as the free energy, the magnetization, the susceptibility, etc. The boundary impurities coupled to the three rapidities affect the low temperature physics at the edges in various different ways, which we now explore.

From the TBA (127), we notice that if $J_{\perp}>J_{c}^{+}=\frac{4 J_{\|}}{\gamma}$ the triplet excitations are massive with energy gap $\Delta=J_{\perp}-\frac{4 J_{\|}}{\gamma}$. The rescaling $\gamma=4$ was fixed [14] for strong coupling compounds, e.g. $\mathrm{Cu}_{2}\left(\mathrm{C}_{5} \mathrm{H}_{12} \mathrm{~N}_{2}\right)_{2} \mathrm{Cl}_{4}[3],\left(\mathrm{C}_{5} \mathrm{H}_{12} \mathrm{~N}\right)_{2} \mathrm{CuBr}_{4}$ [4], etc. Here $J_{c}^{+}$is the critical point at which the quantum phase transition from the three branches of Luttinger liquid to the dimerized $U(1)$ phase occurs. If $J_{\perp}>J_{c}^{+}$, we can show that in the presence of a strong magnetic field two of the triplet states $(|3\rangle$ and $|4\rangle$ in (5)) in the bulk part will never be involved in the ground state. However, at the boundaries this is not always true due to the presence of the boundary impurities. In a strong magnetic field the ground-state may be considered as a condensate of $S U(2)$ hard-core bosons. The gap is reduced by the magnetic field $H$, i.e. $\Delta=J_{\perp}-\frac{4 J_{\|}}{\gamma}-H$. Thus the first critical field occurs at the point $H_{c 1}$ where the gap is closed, i.e. $g \mu_{B} H_{c 1}=J_{\perp}-\frac{4 J_{\|}}{\gamma}$. The quantum phase transition from a gapped phase to gapless Luttinger phase occurs. By continuing to increase the magnetic field $H$ over $H_{c 1}$, the triplet state $|2\rangle$ becomes involved in the ground state with a finite susceptibility, also affected by the boundary impurities in the low concentration regime. If the magnetic field is greater than the rung coupling, i.e. $h>J_{\perp}$, the triplet component $|2\rangle$ becomes the lowest level. Therefore, it is reasonable to choose the basis order as $(|2\rangle,|1\rangle,|3\rangle,|4\rangle)^{\mathrm{T}}$. Subsequently the driving terms are given by $g^{(1)}=-2 \pi J_{\|} a_{1}-J_{\perp}+H, g^{(2)}=J_{\perp}$ and $g^{(3)}=H$. A second critical field $H_{c 2}\left(H_{c 2}>H_{c 1}\right)$ can be determined by the magnetization 
arriving at its saturation value $S^{z}=1$. Then the reference state becomes the true physical state and the critical field $H_{c 2}$ is given by

$$
H_{c 2}=J_{\perp}+\frac{4 J_{\|}}{\gamma}
$$

In this case, all the boundary impurities are gapfull with the ferromagnetic gap $\Delta=\mu_{B} g\left(H-H_{c 2}\right)$.

Let us now discuss the boundary impurity effects in the vicinity of the critical point $H_{c 1}$. After a lengthy calculation, similar to that employed in [14] for the periodic case, we find the free energy in the presence of a strong magnetic field $H$,

$$
\frac{F(0, H)}{2 L} \approx-\frac{4 Q\left(J_{c}^{+}-J_{\mathrm{eff}}\right)}{\pi}\left(1-\frac{2 Q}{\pi}\right)+\frac{1}{2 L} f_{\mathrm{b}}
$$

where $Q$ is the fermi point given by $Q \approx \sqrt{\frac{J_{c}^{+}-J_{\text {eff }}}{4 J_{c}^{+}-5\left(H-H_{c 1}\right)}}$ and $f_{\mathrm{b}}$ is the surface free energy from the boundary impurities in the vicinity of $H_{c 1}$. Explicitly, for $\left|\beta_{ \pm}\right| \geq \frac{1}{2}$, or say $0<U_{ \pm} \leq \frac{J_{\|}}{\gamma}$ or $U_{ \pm}<0$, it is given by

$$
f_{\mathrm{b}} \approx \begin{cases}-\frac{2 Q\left(J_{c}^{+}-J_{\mathrm{eff}}\right)}{\pi}\left(1+\frac{1}{\beta_{+}}+\frac{1}{\beta_{-}}\right), & \text {for } l=m=1,3,5, \\ -\frac{2 Q\left(J_{c}^{+}-J_{\mathrm{eff}}\right)}{\pi}, & \text { for } l=m=2,4 .\end{cases}
$$

For $-\frac{1}{2}<\beta_{ \pm}<<\beta_{c}$, or say $U_{ \pm}>>U_{\mathrm{bs}}=2 J_{\|} / \gamma\left(1-\sqrt{1-\frac{4 J_{\|}}{\gamma J_{\perp}}}\right)$, we have

$$
f_{\mathrm{b}} \approx \begin{cases}-\sum_{ \pm} \frac{2 Q\left(J_{c}^{+}-J_{\mathrm{eff}}\right)}{\pi}\left(\frac{3}{2}+\frac{1}{\beta_{ \pm}}+\frac{1}{\beta_{ \pm}+1}-\frac{1}{\beta_{ \pm}-1}\right) & \\ +\sum_{ \pm}\left(-\frac{J_{\|}}{\gamma} \frac{1}{-\beta_{ \pm}^{2}+\frac{1}{4}}+J_{\perp}\right), & \text { for } l=m=1,3,5 \\ -\frac{2 Q\left(J_{c}^{+}-J_{\mathrm{eff}}\right)}{\pi}, & \text { for } l=m=2,4 .\end{cases}
$$

While for $\left|\beta_{ \pm}\right|$very small, or say $\frac{J_{\|}}{\gamma}<U_{ \pm}<U_{\mathrm{bs}}$, it is given by

$$
f_{\mathrm{b}} \approx \begin{cases}-\frac{2\left(J_{c}^{+}-J_{\mathrm{eff}}\right) Q}{\pi}+\sum_{ \pm} f\left(\beta_{ \pm}\right) & \text {for } l=m=1,3,5, \\ -\frac{2 Q\left(J_{c}^{+}-J_{\mathrm{eff}}\right)}{\pi} & \text { for } l=m=2,4\end{cases}
$$

where 


$$
\begin{aligned}
f\left(\beta_{ \pm}\right)= & -\frac{8 J_{\|}}{\pi \gamma} \frac{1}{1-4 \beta_{ \pm}^{2}}\left(\arctan \frac{Q}{\beta_{ \pm}}-4 Q \beta_{ \pm}\right)+\frac{2 J_{\mathrm{eff}}}{\pi} \arctan \frac{Q}{\beta_{ \pm}} \\
& +\frac{4 Q\left(J_{c}^{+}-J_{\mathrm{eff}}\right)}{\pi(\pi+2 Q)} \frac{1}{1-\beta_{ \pm}^{2}}\left(\arctan \frac{Q}{\beta_{ \pm}}-\beta_{ \pm} Q\right) .
\end{aligned}
$$

In the above $J_{\text {eff }}=J_{\perp}-H$ and the parameters $\beta_{ \pm}$are related to the boundary impurity coupling $U_{ \pm}$by

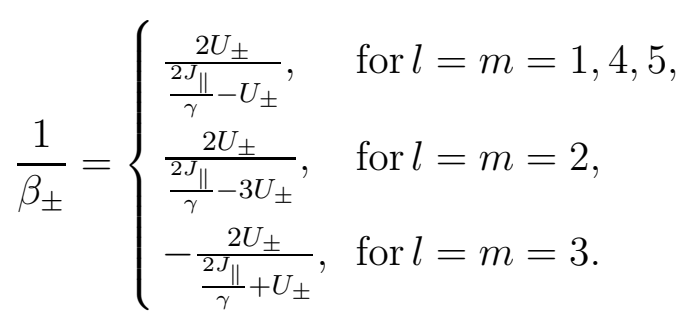

The magnetic susceptibility follows from $\chi \approx-\frac{d^{2}}{d H^{2}} \frac{F(0, H)}{2 L}$. Here, to illustrate the boundary effects, we will focus on the discussion of the strong coupling compounds $J_{\perp}>>J_{\|}$with the boundary impurities in the case $l=m=1$. Other regimes can be handled in a similar way. It is very clear that the stable boundary bound states are exhibited only in the strong ferromagnetic boundary coupling $U_{ \pm}>U_{\text {bs }}$. In Eq.(137), we emphasize that the mathematical singular points do not exist, or alternatively $\beta_{ \pm}=0$ does not mean that the rhs of Eq. (137) has singular points. For instance, if $U_{ \pm}=2 J_{\|} / \gamma$, the boundary parameters $\xi_{ \pm}=1 / 2$. Thus the phase factors in the Bethe ansatz equations (99), (100) and (101) are equal to 1 for the case $l=m=1$. In such a case, the model exihibits special symmetry (the quantum algebra $S U_{q}(4)$ invariant Bethe ansatz equations) which leads to a different expression for the boundary free energy than the above ones. For antiferromagnetic boundary coupling $U_{ \pm}<0$, the susceptibility is given by

$$
\chi \approx \frac{3}{\pi \sqrt{4 J_{c}^{+}\left(H-H_{c 1}\right)}}\left(1+\frac{1}{4 L} \sum_{ \pm}\left(\frac{1}{2}+\frac{1}{\beta_{ \pm}}\right)\right)
$$

while for the strong ferromagnetic coupling $U_{ \pm}>>U_{\mathrm{bs}}$, with $U_{ \pm}>0$,

$$
\chi \approx \frac{3}{\pi \sqrt{4 J_{c}^{+}\left(H-H_{c 1}\right)}}\left(1+\frac{1}{4 L} \sum_{ \pm}\left(\frac{3}{2}+\frac{1}{\beta_{ \pm}}+\frac{1}{\beta_{ \pm}+1}-\frac{1}{\beta_{ \pm}-1}\right)\right) .
$$

Notice, in both cases, that the susceptibility diverges with the square root of the field in the bulk and in the boundaries. In addition, the susceptibility at the boundaries is enhanced or decreased by different impurity dopings. This behaviour is illustrated in figure 2. From the Bethe ansatz equations, we can 


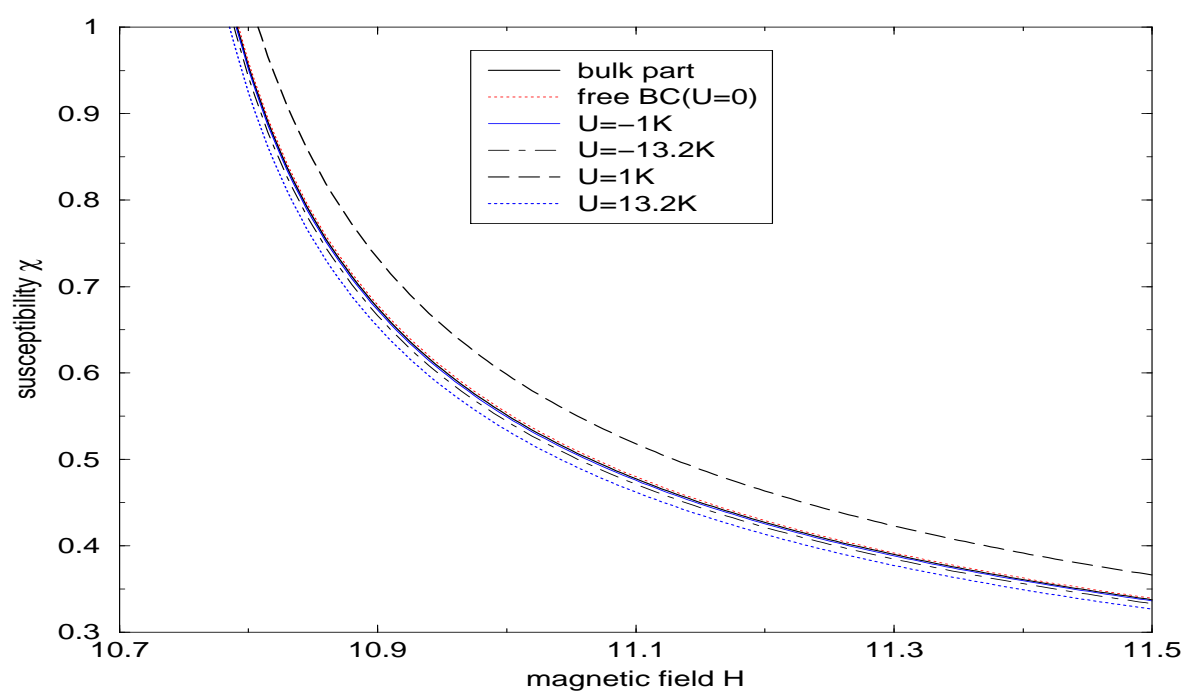

Fig. 2. The susceptibility versus magnetic $H$ for different impurity coupling $U_{ \pm}=U$ ( $U=0$ corresponds to free boundary conditions). Here we consider the strong coupling compound $\mathrm{Cu}_{2}\left(\mathrm{C}_{5} \mathrm{H}_{12} \mathrm{~N}_{2}\right)_{2} \mathrm{Cl}_{4}[3]$ with $J_{\perp}=13.2 \mathrm{~K}, J_{\|}=2.5 \mathrm{~K}$ and $\gamma=4$ with an impurity concentration 2 percent in a ladder with length $L=50$.

also calculate the magnetization in the vicinity of $H_{c 1}$,

$$
\frac{S^{z}}{2 L}=\int_{-Q}^{Q} \rho_{1}^{(1)}(\lambda) d \lambda=\frac{4 Q}{\pi}\left(1-\frac{2 Q}{\pi}\right)+\frac{1}{2 L} \sum_{ \pm} S_{\mathrm{b}}^{z} .
$$

For antiferromagnetic boundary coupling $U_{ \pm}<0$ this expression reduces to

$$
S_{\mathrm{b}}^{z} \approx \sum_{ \pm} \frac{2 Q}{\pi}\left(1-\frac{2 Q}{\pi}\right)\left(\frac{1}{2}+\frac{1}{\beta_{ \pm}}\right)
$$

while for strong ferromagnetic boundary coupling $U_{ \pm}>>U_{\mathrm{bs}}$ with $U_{ \pm}>0$,

$$
S_{\mathrm{b}}^{z} \approx \sum_{ \pm} \frac{2 Q}{\pi}\left(1-\frac{2 Q}{\pi}\right)\left(\frac{3}{2}+\frac{1}{\beta_{ \pm}}+\frac{1}{\beta_{ \pm}+1}-\frac{1}{\beta_{ \pm}-1}\right) .
$$

A plot of the magnetization $S^{z}$ against the magnetic field for different boundary impurities $U_{ \pm}$is given in figure 3 . By analyzing both figures we can observe the competition between the boundary impurities and the magnetic field in the thermodynamic properties. In particular, we find an enhancement of the susceptibility in the weak anti- and ferromagnetic regimes (we consider the sizes $2 L=100$, the impurity concentration 2 percent ). The susceptibility and the magnetization are lifted slightly in the weak antiferromagnetic boundary regime in the case of open boundary conditions, whereas they contribute 


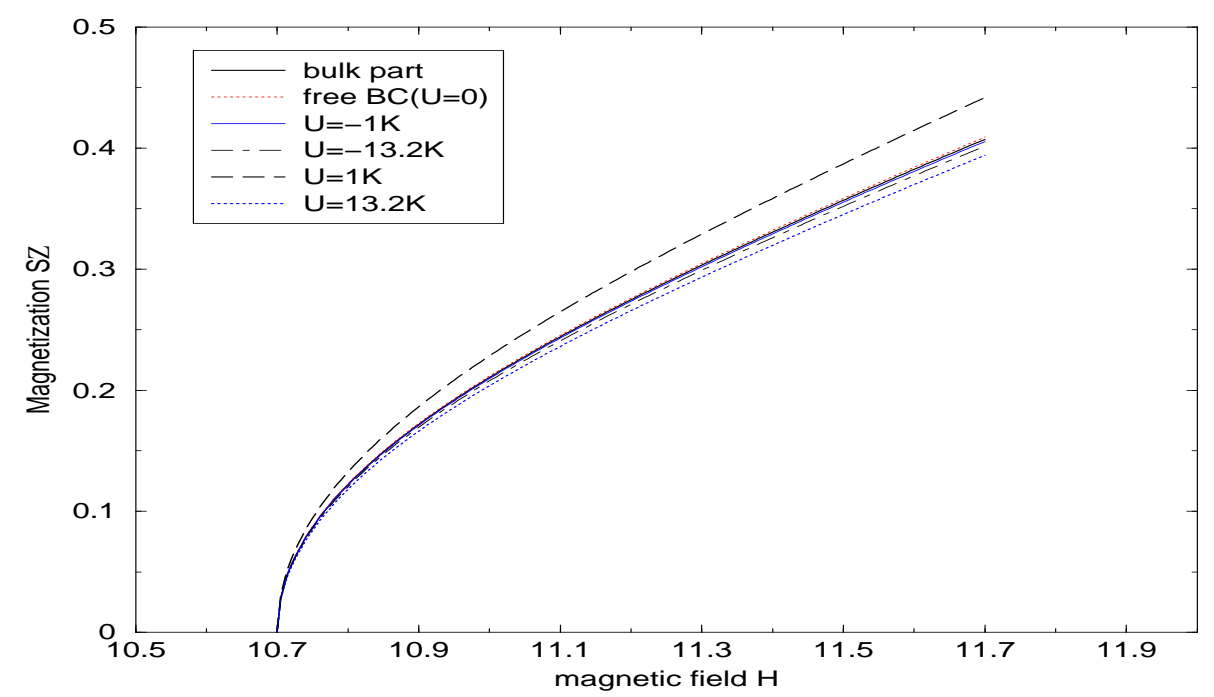

Fig. 3. The magnetization versus magnetic field $H$ for different impurity coupling $U_{ \pm}=U(U=0$ corresponds to free boundary conditions). As in figure 2 , we consider the strong coupling compound $\mathrm{Cu}_{2}\left(\mathrm{C}_{5} \mathrm{H}_{12} \mathrm{~N}_{2}\right)_{2} \mathrm{Cl}_{4}$ [3] with $J_{\perp}=13.2 \mathrm{~K}, J_{\|}=2.5 \mathrm{~K}$ and $\gamma=4$ with an impurity concentration 2 percent in a ladder with length $L=50$.

negatively to the bulk when $U_{ \pm}$becomes more and more negative. This is reasonable, since negative $U_{ \pm}$energetically favours the singlet state (recall the boundary terms in the Hamiltonian (1)), even if the magnetic field is very strong, such that the spin-1 component of the triplet is involved in the ground state. The point is that a very negative $U_{ \pm}$can overcome the spin- 1 component of the triplet and dominate the edge rung state. In this circumstance, the edge state is a pure singlet state and the edge magnetization (141) is zero due to the fact that $U_{ \pm}$effectively decreases the edge magnetic field $H$ to $H_{c 1}$ such that the fermi boundary $Q=0$. This results in negative susceptibility and magnetization contributions to the bulk. In contrast to this case, the ferromagnetic impurities lift the susceptibility and the magnetization in the weak coupling regime $U_{ \pm}<\frac{J_{\|}}{\gamma}$. When $U_{ \pm}$becomes larger, the triplet edge state is energetically favoured so that the boundary coupling can overcome the magnetic field to bring the three components of the triplet into the edge states. Therefore it causes a negative contribution to the bulk susceptibility and magnetization. This situation is different from the case of the bulk impurities, where the susceptibility is increased by the impurity coupling due to the forward-scattering. This fact can be seen clearly from the one point correlation function of the ground state at the edges, for antiferromagnetic boundary coupling and weak ferromagnetic boundary coupling, i.e. $U_{ \pm} \leq \frac{J_{\|}}{\gamma}$,

$$
\left\langle\vec{S}_{a} \cdot \vec{T}_{a}\right\rangle=-\frac{3}{4}+\frac{d}{d U_{ \pm}} f_{\mathrm{b}}=-\frac{3}{4}+\frac{2 Q\left(H-H_{c 1}\right)}{\pi} \frac{4 J_{\|} / \gamma}{\left(2 J_{\|} / \gamma-U_{ \pm}\right)^{2}}
$$

and for ferromagnetic impurities in the strong coupling regime $U_{ \pm}>>P_{\mathrm{bs}}$, 


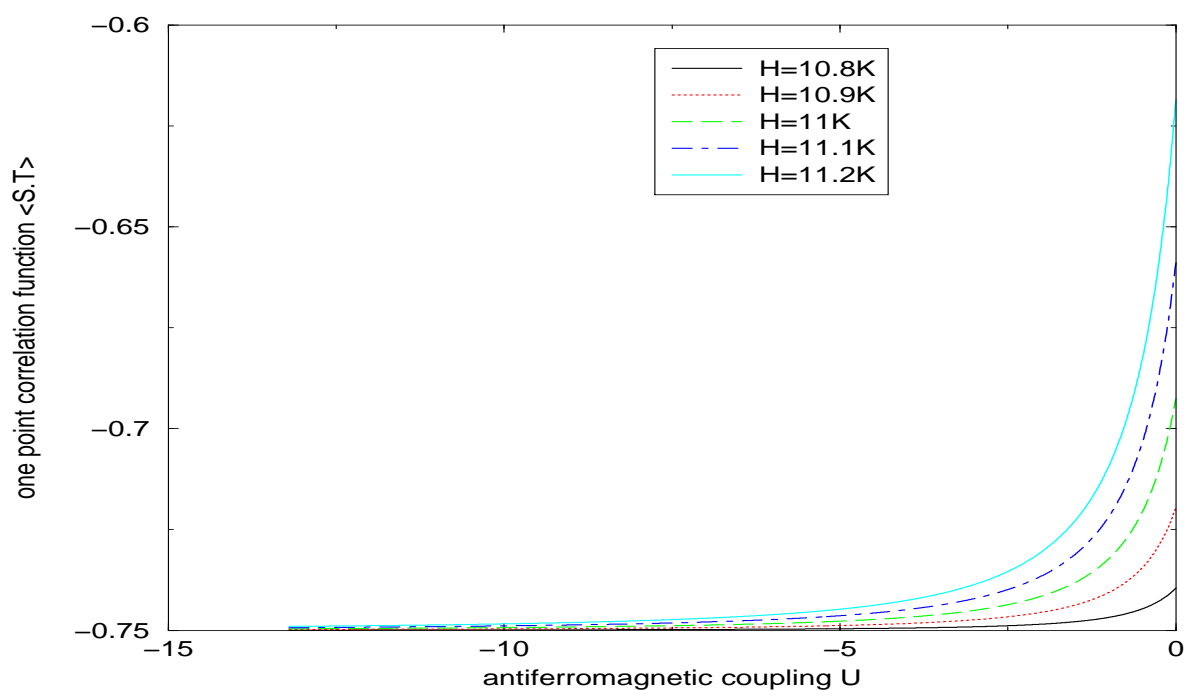

Fig. 4. One point correlation function (143) versus antiferromagnetic boundary coupling $U$ as a function of magnetic field. The curve is lifted by the magnetic field, however it is decreased by the boundary impurities which favour the singlet state. Here we consider the strong coupling compound $\mathrm{Cu}_{2}\left(\mathrm{C}_{5} \mathrm{H}_{12} \mathrm{~N}_{2}\right)_{2} \mathrm{Cl}_{4}[3]$ with $J_{\perp}=13.2 \mathrm{~K}$, $J_{\|}=2.5 K$ and $\gamma=4$ and $U_{ \pm}=U$. The case $U=0$ corresponds to the free boundary effect.

$$
\begin{aligned}
\left\langle\vec{S}_{a} \cdot \vec{T}_{a}\right\rangle= & \frac{1}{4}+\frac{2 Q\left(H-H_{c 1}\right)}{\pi}\left[\frac{4 J_{\|} / \gamma}{\left(2 J_{\|} / \gamma-U_{ \pm}\right)^{2}}\right. \\
& \left.-\frac{4 J_{\|} / \gamma}{\left(2 J_{\|} / \gamma-3 U_{ \pm}\right)^{2}}+\frac{4 J_{\|} / \gamma}{\left(2 J_{\|} / \gamma+U_{ \pm}\right)^{2}}\right]-\frac{1}{\left(1-\frac{\gamma U_{ \pm}}{J_{\|}}\right)^{2}} .
\end{aligned}
$$

In the above $a=1, L$. The boundary one point correlation functions are given by

$$
\left\langle\vec{S}_{a} \cdot \vec{T}_{a}\right\rangle=-\frac{3}{4}\left\langle N_{\mathrm{S}}\right\rangle+\frac{1}{4}\left\langle N_{\mathrm{T}}\right\rangle
$$

Here $N_{\mathrm{S}}$ and $N_{\mathrm{T}}$ are the probabilities of the singlet and triplet state respectively. This is because the eigenvalue of the one point correlation function $\left\langle\vec{S}_{a} \cdot \vec{T}_{a}\right\rangle$ acting on the singlet (triplet) state is $-\frac{3}{4}\left(\frac{1}{4}\right)$. We have plotted the correlation function for antiferromagnetic boundary coupling in figure 4.

We see that the magnetic field always lifts the spin- 1 triplet component. However, in the case of antiferromagnetic boundary impurities and open boundaries the singlet state is favoured as long as $U_{ \pm}$becomes more negative, the triplet moves out of the edge state and the one point correlation function tends to $-\frac{3}{4}$. On the other hand, for ferromagnetic coupling impurities (see figure 5), 
(a)

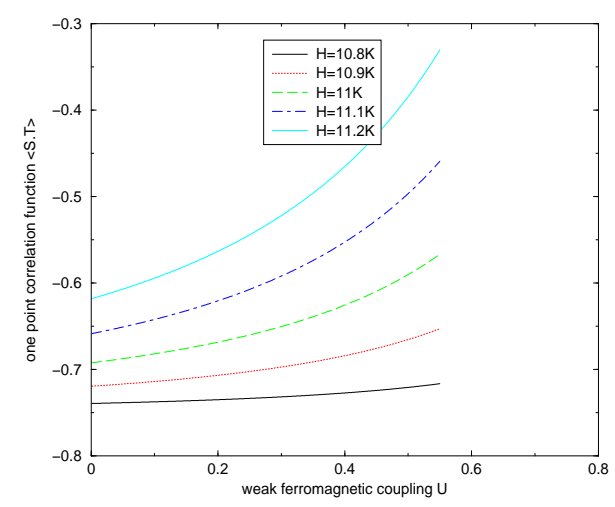

(b)

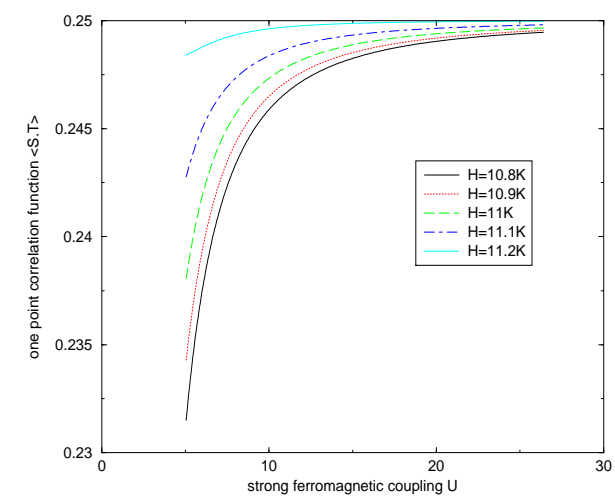

Fig. 5. One point correlation function vs ferromegnetic boundary coupling $U$ :(a) The function (143) is lifted by the magnetic field and weak magnetic impurity coupling $U$. (b) The function (144) tends to $\frac{1}{4}$ as the boundary impurity coupling becomes larger. Here we again consider the strong coupling compound $\mathrm{Cu}_{2}\left(\mathrm{C}_{5} \mathrm{H}_{12} \mathrm{~N}_{2}\right)_{2} \mathrm{Cl}_{4}$ [3] with $J_{\perp}=13.2 \mathrm{~K}, J_{\|}=2.5 \mathrm{~K}$ and $\gamma=4$ and $U_{ \pm}=U$. The case $U=0$ corresponds to free boundaries.

the correlation function increases due to the ferromagnetic properties and the magnetic field in the weak coupling regime $U_{ \pm}<\frac{J_{\|}}{\gamma}$. However, if $U_{ \pm}$becomes larger, the three components of the triplet get involved in the edge state, such that the correlation function tends to $\frac{1}{4}$ for strong ferromagneitc impurity coupling. This result indicates that the edge state can be a pure singlet state in the strong antiferromagnetic boundary coupling regime whereas it turns out to be a pure triplet state in the strong ferromagnetic boundary coupling regime. This reveals the role of anti- and ferromagnetic impurities.

On the other hand, the boundary impurities coupled to the spin degrees of freedom, namely, $\rho_{\mathrm{b}}^{(2)}$ and $\rho_{\mathrm{b}}^{(3)}$ will also affect the ground state properties nontrivially. From the free energy (128), these impurity densities will contribute to the low energy. Considering the case $J_{\perp}<0$, in the absence of the magnetic field, the triplet is completely degenerate while the fermi surface of the singlet is lifted as $J_{\perp}$ becomes more negative. Certainly, if $J_{\perp}<J_{c}^{-}=-\frac{J_{\|}}{\gamma}\left(\frac{\pi}{\sqrt{3}}-\ln 3\right)$ the singlet rung state is not involved in the ground-state, namely $\epsilon^{(3)}(0) \geq 0$, whereas two triplet fermi seas still have fermi boundaries at infinity. Under such a configuration, the dressed energy potentials are

$$
\epsilon^{(1)}(\lambda)=-\frac{2 \pi J_{\|}}{\sqrt{3} \gamma} \frac{\cosh \frac{\pi}{3} \lambda}{\cosh \pi \lambda}, \quad \epsilon^{(2)}(\lambda)=-\frac{2 \pi J_{\|}}{\sqrt{3} \gamma} \frac{\sinh \frac{\pi}{3} \lambda}{\sinh \pi \lambda} .
$$


The free energy can be given by

$$
\frac{F(0,0)}{2 L} \approx-\frac{2 J_{\|}}{3 \gamma}\left(\psi(1)-\psi\left(\frac{1}{3}\right)\right)+\frac{1}{2 L} f_{\mathrm{b}}
$$

where

$$
f_{\mathrm{b}}=\int_{-\infty}^{\infty} \rho_{\mathrm{b} 1}^{(1)}(\lambda) \epsilon_{1}^{(1)}(\lambda) \mathrm{d} \lambda+\int_{-\infty}^{\infty} \rho_{\mathrm{b} 1}^{(2)}(\lambda) \epsilon_{1}^{(2)}(\lambda) \mathrm{d} \lambda
$$

The first part in (147) is nothing but the standard $S U(3)$ ground state energy of the bulk. The remaining part is the boundary surface energy for various boundary impurities.

\section{Conclusion and discussion}

In summary, we have discussed in detail the algebraic Bethe-ansatz solution of an integrable spin ladder system based on the $S U(4)$ symmetry with boundary impurities. Five different classes of solutions of the graded RE leading to different boundary rung interactions in the Hamiltonian were obtained. The Bethe-ansatz equations, the eigenvalues of the transfer matrix and the energy spectrum were given explicitly. Furthermore, the three-level transfer matrices, characterizing the different flavour sectors separately, allowed us to embed different impurities into the system. From the Bethe ansatz solutions (112)-(114), we found that the boundary impurity effects characterized by $\zeta\left(v_{i}, \xi_{ \pm}\right), \eta\left(\mu_{j}, \xi_{ \pm}\right)$and $\Omega\left(w_{k}, \xi_{ \pm}\right)$act indeed non trivially on the densities of roots for the three rapidities and thus change the ground state properties, the boundary bound states as well as the low-lying energy spectrum. In the thermodynamic limit, the spin gap remains almost unchanged. However, the boundary susceptitblity and magnetization reveal novel magnetic properties for strong and weak impurity couplings. In strong impurity coupling, the impurities induced by the open boundary conditions can result in either a pure triplet or a singlet edge state due to the nature of the pure back-scattering at the edges and the magnetic impurities. Strictly speaking, the edge state can be a pure singlet state in a strong antiferromagnetic boundary coupling regime whereas a triplet state with an effective magnetic moment can exist in a strong ferromagnetic boundary coupling regime. Correspondingly, the one point correlation function for strong antiferromagnetic boundary impurities tends to

the singlet eigenvalue $-\frac{3}{4}$, whereas for strong ferromagneitc impurity coupling it tends to the triplet eigenvalue $\frac{1}{4}$. This behaviour may be observed in experiments due to different boundary magnetic moments. Although the TBA solution of the $S U(4)$ ladder model (1) predicts the quantum phase diagram in 
good agreement with experimental results for the strong coupling compounds, the full finite temperature thermodynamic properties of the model remain to be calculated.

\section{Acknowledgements}

A.F. and X.W.G. would like to thank J. Links, I. Roditi, R.A. Römer and Z.-J. Ying for helpful discussions. M.T.B., X.W.G. and H.Q.Z thank the Australian Research Council for financial support. A.F. and A.P.T. thank CNPq and FAPERGS for financial support.

\section{References}

[1] E. Dagotto and T.M. Rice, Science 271 (1996) 618; E. Dagotto, Rep. Prog. Phys. 62 (1999) 1525.

[2] M. Azuma, Z. Hiroi, M. Takano, K. Ishida and Y. Kitaoka, Phys. Rev. Lett. 73 (1994) 3463.

[3] G. Chaboussant, et al, Phys. Rev. Lett. 79 (1997) 925;

G. Chaboussant, et al, Phys. Rev. Lett. 80 (1998) 2713;

G. Chaboussant, P.A. Crowell, L.P. L evy, O. Piovesana, A. Madouri and D. Mailly, Phys. Rev. B55 (1997) 3046.

[4] B.C. Watson, V.N. Kotov and M.W. Meisel, Phys. Rev. Lett. 86 (2001) 5168.

[5] C.P. Landee, M.M. Turnbull, C. Galeriu, J. Giantsidis and F.M. Woodard, cond-mat/0011016.

[6] W. Shiramura, et al, J. Phys. Soc. Japan 66 (1997) 1900.

[7] A. A. Nersesyan and A. M. Tsvelik, Phys. Rev. Lett. 78 (1997) 3939.

[8] A. K. Kolezhuk and H.-J Mikeska, Int. J. Mod. Phys. B 12 (1998) 2325; Phys. Rev. Lett. 80 (1998) 2709.

[9] P.W. Anderson, Science 235 (1987) 1196;

F.C. Zhang and T.M. Rice, Phys. Rev. B 37 (1988) 3759.

[10] H. Frahm and A. Kundu, J. Phys. C: Cond. Mat. 11 (1999) L557.

[11] M. T. Batchelor and M. Maslen, J. Phys. A 32 (1999) L377; J. Phys. A 33 (2000) 443;

M.T. Batchelor, J. de Gier and M. Maslen, J Stat. Phys. 102 (2001) 559; Phys. Rev. B 61 (2000) 15196.

J. de Gier and M.T. Batchelor, Phys. Rev. B 62 (2000) R3584. 
[12] Y. Wang, Phys. Rev. B 60 (1999) 9236.

[13] J. Links and A. Foerster, Phys. Rev. B62 (2000) 3845;

A. Foerster, K.E. Hibberd, J.R. Links and I. Roditi, J. Phys. A 34 (2001) L25.

[14] M.T. Batchelor, X.-W. Guan, A. Foerster and H.-Q. Zhou, Quantum phase diagram of strong coupling ladder compounds from thermodynamic Bethe ansatz, preprint.

[15] Y. Wang and P. Schlottmann, Phys. Rev. B62 (2000) 3845.

[16] I.V. Cherednik, Theor. Math. Phys. 61 (1984) 977.

[17] E.K. Sklyanin, J. Phys. A: Math. Gen. 21 (1988) 2375;

L. Mezincescu and R.I. Nepomechie, Int. J. Mod. Phys. A6 (1991) 5231; 7 (1992) 5657 .

[18] H.J. de Vega and A. Gonzalez Ruiz, J. Phys. A: Math. Gen. 26 (1993) L519; M.T. Batchelor, V. Frikin, A. Kuniba and Y.K. Zhou, Phys. Lett. B 376 (1996) 266.

[19] A.J. Bracken, X.Y. Ge, Y.Z. Zhang and H.Q. Zhou, Nucl. Phys. B 516 (1998) 588 ;

H.Q. Zhou, Phys. Rev. B 53 (1996) 5089; Phys. Lett. A 228 (1997) 48.

[20] M.J. Martins, X.-W. Guan, Nucl. Phys. B 583 (2000) 721.

Xi-Wen Guan, J. Phys. A. 33 (2000) 5391;

M. Shiroishi and M. Wadati, J. Phys. Soc. Jpn. 66 (1997) 2288.

[21] Y. Motome, N. Katoh, N. Furukawa and M. Imada, J. Phys. So. Japan, 65 (1996) 1949, and references therein.

[22] A. Foerster and M. Karowski, Nucl. Phys. B 396 (1993) 611.

[23] M.J. Martins and P.B. Ramos, Nucl. Phys. B 522 (1998) 413.

[24] X.-W. Guan, A. Foerster, U. Grimm, R.A. Römer and M. Schreiber, Nucl. Phys. B 618 (2001) 650.

[25] A. Foerster, X.-W. Guan, J. Links, I. Roditi and H.-Q. Zhou, Nucl. Phys. B 596 (2001) 525 .

[26] M. Takahashi, Prog. Theor. Phys. 46 (1971) 401; P. Schlottmann, Phys. Rev. B33 (1986) 4880.

[27] H.M. Babujian, Nucl. Phys. B 215 (1983) 317.

[28] C.N. Yang and C.P. Yang, Phys. Rev. B 150 (1966)321; B 150 (1966) 327. N. Fukushima and Y. Kuramoto, J. Phys. Soci. Japan 71 (2002) 1238. 